\title{
Leaf flushing behaviour of teak clones of Madhya Pradesh, India
}

\author{
N. Roychoudhury, Manish Chourasia and Rajesh Kumar Mishra*
}

ICFRE-Tropical Forest Research Institute, P.O. RFRC, Mandla Road, Jabalpur-482021, Madhya Pradesh, India

*Corresponding Author: rajeshkmishra20@gmail.com

\begin{abstract}
The leaf flushing period as a parameter of morphological trait of its behaviour was studied for three years (2002-2004) in 123 teak clones belonging to eight Forest Divisions (Balaghat, Betul, Chhindwada, Hardha, Hoshangabad, Khandwa, Mandla and Seoni) of Madhya Pradesh, planted in ten experimental plots at teak seed orchard (TSO), Behrai, Seoni, Madhya Pradesh, India. The observations revealed that leaf flushing in teak clones of Madhya Pradesh occur in the month of May-June, irrespective of clones and years. Out of 123 teak clones, 18 clones showed early leaf flushing $\left(16^{\text {th }}\right.$ May to $31^{\text {st }}$ May), 78 clones exhibited mid leaf flushing ( $1^{\text {st }}$ June to $15^{\text {th }}$ June) and 27 clones revealed late leaf flushing ( $16^{\text {th }}$ June to $30^{\text {th }}$ June). The variation in leaf flushing periods within the clones and among the clones of different localities were found to be significantly $(\mathrm{P}<0.05$ to $\mathrm{P}<0.01)$ heterogeneous within and over the years. The findings advocate that the variation in leaf flushing period of clones may be due to difference in genotypes of teak.
\end{abstract}

Keywords: Teak, Clones, Leaf flushing period, Madhya Pradesh.

\section{INTRODUCTION}

Teak (Tectona grandis L.f.) (family Verbenaceae), is an undisputed global leader of high-quality tropical timbers. It is referred to as standard timber for comparative evaluation of the qualities of other tropical hardwoods in assessing their utilization potential (Bhat et al., 2005). Teak is truly an Indian species because the Indian region is considered to be the only known centre of maximum genetic diversity and variability of teak (Hedegart, 1975) with distribution over 8.9 million hectare (Seth \& Kaul, 1978). The natural teak forests of India are confined to Peninsular India below $24^{\circ} \mathrm{N}$ latitude with a total area of about 1.4 million hectare (Pande, 1983). The major teak growing states are Kerala, Karnataka, Tamilnadu, Andhra Pradesh, Madhya Pradesh, Chhattisgarh, Maharashtra, Orissa, Uttar Pradesh, Gujarat and Rajasthan. The most important teak forests, however, occur in Wynad of Kerala, North Kanara of Karnataka, Anamalai hills of Tamilnadu, Hoshangabad and Betul of Madhya Pardesh and Chanda and Melghat of Maharashtra (Kumaravelu, 1992).

Madhya Pradesh, the heartland of India, is one of the most important states with extensive teak forests, highest number of superior teak clones, teak seed orchards (TSOs) both clonal seed orchards (CSOs) and seedling seed orchards (SSOs) and famous for its high-quality wood (Tiwari et al., 1998a, b; Roychoudhury et al., 2003). It has a geographical area of 308 lakh $\mathrm{km}^{2}$ and, the largest forest area of $95,221 \mathrm{~km}^{2}$ and the highest forest cover of 77,265 km with a growing stock of 50 million $\mathrm{m}^{3}$. Teak occurs in over $20 \%$ of forest area, which is predominantly distributed in the southern and central parts of Madhya Pradesh (Gangopadhyay, 2005). The rich teak forests are found in the districts of Hoshangabad, Harda, Betul, Chhindwara, Seoni, Mandla and Balaghat. Teak forest of relatively lower site quality is also found in Panna, Sagar, Raisen, Vidisha, Sehore, Dewas, Guna and Khandwa districts. This state has also 377 superior phenotypic trees (plus trees) of teak which have been identified from the 16 Forest Divisions of Madhya Pradesh namely Hoshangabad, Harda, North Betul, South Betul, Khandwa, East Chhindwara, South Seoni, South Balaghat, East Mandla, West Mandla, Dewas, Indore, Jhabua, Damoh, Sagar and Bhopal, and an area of 358 ha CSOs and 186 ha SSOs established in different eco-zones of Madhya Pradesh (Shukla et al., 2003; Roychoudhury, 2016). Apart from the preliminary information about the leaf flushing behaviour in teak (Priya \& Baht, 1998; Ansari et al., 2001), no such work has been carried out in teak clones. The present paper is an attempt to study in detail the leaf flushing behaviour of 123 superior teak clones of Madhya Pradesh origin.

\section{MATERIALS AND METHODS}

\section{Study materials}

Teak clones of Madhya Pradesh origin planted in teak seed orchard (TSO) located at Ghisi, Behrai, Seoni, Madhya Pradesh, India, were considered as a source of study materials. This seed orchard is an assemblage of 123 superior teak clones collected from 8 Forest Divisions of Madhya Pradesh, viz., Balaghat, Betul, Chhindwada, Hardha, Hoshangabad, Khandwa, Mandla and Seoni (Table 1) and planted in compartment No 54, with randomized block design and $8 \times 8 \mathrm{~m}$ spacing in 10 plots, during the year 1977-1985. This is the largest TSO in Madhya Pradesh, India, with a total area of 50 
ha, which lies between $79^{\circ} 19^{\prime}$ and $80^{\circ} 17^{\prime} \mathrm{E}$ longitude, $21^{\circ} 11^{\prime}$ and $22^{\circ} 57^{\prime} \mathrm{N}$ latitude and at an altitude of $619 \mathrm{~m}$ above the mean sea level. The mean maximum and minimum temperature are $43.4^{\circ} \mathrm{C}$ and $5.6^{\circ} \mathrm{C}$ respectively with an annual rainfall of $1200 \mathrm{~mm}$. The plantation details and a total of 6874 grafts of $123 \mathrm{MP}$ teak clones planted in TSO, Behrai, Seoni, during the different years are presented in tables 2 and 3 respectively.

Table 1. Teak clones of Madhya Pradesh origin planted in TSO, Behrai, Seoni, Madhya Pradesh.

\begin{tabular}{llllr}
\hline $\begin{array}{l}\text { Forest } \\
\text { Division }\end{array}$ & $\begin{array}{l}\text { MP teak } \\
\text { (Code) }\end{array}$ & $\begin{array}{l}\text { Locality from where } \\
\text { plus tree selected }\end{array}$ & Clone Number & Total \\
\hline Balaghat & BLC & Balaghat Lanjhi & BLC-1, 5, 6, 7, 8, 10, 11 & 7 \\
Betul & G & Betul & G-1 & 1 \\
& F & Betul & F-1 & 1 \\
& BBC & Betul Baretha & BBC-12, 14, 15 & 3 \\
Chhindwada & CSC & Chhindwada & CSC-1, 4, 5, 6, 7, 8, 9, 10, 11, 12, 13, 14, 15, 16, 17, 18, & 31 \\
& & Sillewani & $19,20,21,22,23,24,25,26,27,28,29,30,31,32,60$ & 1 \\
Hardha & B & Hardha & B-1 & 4 \\
Hoshangabad & BHC & Bori Hoshangabad & BHC-16, 17, 18, 19 & 1 \\
Khandwa & K & Khandwa & K-1 & 1 \\
Mandla & M & Mandla & M-1 & 1 \\
& MBC & Mandla Barela & MBC-4 & 2 \\
& MKC & Mandla Kalpi & MKC-24, 25 & 5 \\
Seoni & MTC & Mandla Tikaria & MTC- 6, 7, 8, 12, 27 & 5 \\
& C & Seoni Kurai & C-1, 2, 3, 4, 5, 6, 7, 8, 9, 10, 11, 12, 14, 15, 16, 17, 18, 19, & 55 \\
& & & $20,22,23,24,25,26,27,28,29,30,31,32,33,34,35$, & \\
& & & $36,37,38,39,40,41,42,44,45,46,47,48,49,50,51$, & \\
\hline
\end{tabular}

Table 2. Plantation details of TSO, Behrai, Seoni, Madhya Pradesh*.

\begin{tabular}{lrrrr}
\hline Establishment Year & Area (ha) & No. of grafts planted & No. of Clone & Plot No. \\
\hline 1977 Part I & 3.68 & 505 & 19 & I \\
1978 Part I & 1.45 & 220 & 9 & II \\
1979 Part I & 7.31 & 986 & 42 & III \\
1979 Part II & 1.84 & 250 & 16 & IV \\
1980 Part I & 10.36 & 1575 & 46 & V \\
1980 Part II & 1.22 & 192 & 12 & VI \\
1981 Part I & 8.00 & 1221 & 48 & VII \\
1981 Part II & 1.20 & 314 & 15 & VIII \\
1981 Part III** & 0.77 & 99 & 24 & \\
1982 Part I & 8.09 & 1445 & 37 & IX \\
1985 Part I & 0.96 & $154 * * *$ & 20 & X \\
1985 Part II & 1.61 & $218 * * *$ & 17 & X \\
Total & $\mathbf{4 6 . 4 9}$ & $\mathbf{7 1 7 9}$ & $\mathbf{3 0 5}$ & \\
\hline Note: *Total area $=$ 50 ha; $*$ National provenance trial; ***206 grafts belongs & to \\
Maharashtra Compartment No. 54; Spacing between ramets $=8$ m. & &
\end{tabular}

Table 3. Number of grafts of different MP teak clones planted in TSO, Behrai, Seoni, in different years.

\begin{tabular}{|c|c|c|c|c|c|c|c|c|c|c|c|}
\hline \multirow{3}{*}{$\begin{array}{l}\text { MP } \\
\text { teak clone* }\end{array}$} & \multicolumn{10}{|c|}{ Year of Establishment } & \multirow{3}{*}{ Total } \\
\hline & \multirow{2}{*}{1977} & \multirow{2}{*}{1978} & \multicolumn{2}{|c|}{1979} & \multicolumn{2}{|c|}{1980} & \multicolumn{2}{|c|}{1981} & \multirow{2}{*}{1982} & \multirow{2}{*}{$\begin{array}{r}1985 \\
\text { I \& II }\end{array}$} & \\
\hline & & & I & II & I & II & I & II & & & \\
\hline B-1 & 17 & & & & & & & & & & 17 \\
\hline $\mathrm{C}-1$ & 30 & & 12 & & & & 50 & 15 & & 13 & 120 \\
\hline $\mathrm{C}-2$ & 44 & & 15 & & & & 40 & & & & 99 \\
\hline $\mathrm{C}-3$ & 20 & 35 & 14 & & & & & & & & 69 \\
\hline C-4 & & 10 & & & 35 & & 50 & 20 & & & 115 \\
\hline C-5 & & 10 & 15 & & & & 15 & & & & 40 \\
\hline C-6 & 46 & & & & & & 10 & & 56 & & 112 \\
\hline $\mathrm{C}-7$ & & & & & & & & & 47 & & 47 \\
\hline $\mathrm{C}-8$ & 20 & & 40 & 12 & 33 & & & & 56 & 15 & 176 \\
\hline C-9 & 20 & & & & 35 & & & & 44 & & 99 \\
\hline $\mathrm{C}-10$ & 20 & & & & 35 & & & & 12 & & 67 \\
\hline $\mathrm{C}-11$ & 43 & 10 & & & 35 & & & & 49 & & 137 \\
\hline $\mathrm{C}-12$ & 15 & & & & & & & & 56 & & 71 \\
\hline
\end{tabular}


Plants and Environment (2020) 2(1): 13-30

\begin{tabular}{|c|c|c|c|c|c|c|c|c|c|c|c|}
\hline C-14 & & & & & & & & & 12 & & 12 \\
\hline C- 15 & & 25 & 40 & & & & & & 56 & & 121 \\
\hline C-16 & & & & & & & & & 12 & & 12 \\
\hline C-17 & & & 25 & & & & & & 12 & 13 & 50 \\
\hline C-18 & & & & & & & 10 & & 17 & & 27 \\
\hline C-19 & & & 30 & & & & 30 & & & & 60 \\
\hline C-20 & & & 30 & & & & & & & & 30 \\
\hline C-22 & & & 40 & 18 & & & & & & & 58 \\
\hline C-23 & & & 15 & & & & & & & & 15 \\
\hline C-24 & & & 30 & & & & & & & & 30 \\
\hline C-25 & & & 15 & 12 & 35 & & 11 & & & & 73 \\
\hline C-26 & & & 30 & 10 & 30 & & & & 10 & 12 & 92 \\
\hline C-27 & & & 30 & 18 & 35 & & & & & & 83 \\
\hline C- 28 & & & 35 & 18 & & 16 & & & & & 69 \\
\hline C-29 & & & & & & 16 & & & & & 16 \\
\hline C-30 & & & & & & & & & 39 & & 39 \\
\hline C-31 & & & & & & & & 12 & & & 12 \\
\hline C-32 & & & & & & 16 & & & & & 16 \\
\hline C-33 & & & & & & & & & 47 & & 47 \\
\hline C-34 & & & 25 & & & & 50 & 42 & & 13 & 130 \\
\hline C-35 & & & 35 & 18 & & 16 & & & & & 69 \\
\hline C-36 & & & & & & & 11 & & & & 11 \\
\hline C-37 & & & 35 & & & & & & & & 35 \\
\hline C-38 & & & & 18 & 35 & & & & & & 53 \\
\hline C-39 & & & 30 & 18 & 35 & & & & & & 83 \\
\hline$C-40$ & & & 30 & 18 & 35 & & 50 & & & & 133 \\
\hline C-41 & & & 18 & & 35 & & 30 & & & & 83 \\
\hline C- 42 & & & 18 & & & & 50 & 46 & 56 & & 170 \\
\hline C-44 & & & 13 & 10 & & & & & & & 23 \\
\hline C-45 & & & 30 & 18 & 35 & 16 & 50 & 13 & & & 162 \\
\hline C-46 & & & 30 & 14 & & & & & & 12 & 56 \\
\hline C-47 & & & 30 & & & & 50 & 12 & & 13 & 105 \\
\hline C- 48 & & & 25 & & & & 10 & & & & 35 \\
\hline C-49 & & & 30 & & 30 & & 20 & & & 12 & 92 \\
\hline C-50 & & & 33 & & 42 & & & & & & 75 \\
\hline C-51 & & & 27 & & & & & & & & 27 \\
\hline C-52 & & & 31 & 18 & & & & & & & 49 \\
\hline C-53 & & & & & & & & & & 12 & 12 \\
\hline C-54 & & & 11 & 12 & & & & & & & 23 \\
\hline C-56 & & & 11 & 18 & & & 50 & & & & 79 \\
\hline C-57 & & & 15 & & 30 & & 10 & & & & 55 \\
\hline C-59 & & & & & 35 & & & & 52 & & 87 \\
\hline C-60 & & & 11 & & 30 & & & & & & 41 \\
\hline F-1 & 20 & & 11 & & 35 & & & & & & 66 \\
\hline G-1 & 16 & & & & & & & & & & 16 \\
\hline K-1 & 16 & & & & 35 & & & & & & 51 \\
\hline M-1 & & & & & 12 & & & & & & 12 \\
\hline PT-1 & 21 & 25 & & & 35 & 16 & 50 & 40 & & 14 & 201 \\
\hline PT-3 & 43 & & & & & & & & 54 & 11 & 108 \\
\hline PT-5 & & & & & & & 30 & & & & 30 \\
\hline PT-6 & & 35 & & & & & 20 & & & & 55 \\
\hline PT-7 & & & & & & & 50 & 20 & & & 70 \\
\hline PT-26 & 26 & & & & & & & & & & 26 \\
\hline PT-41 & 27 & & & & & & & & & & 27 \\
\hline PT-45 & 35 & 35 & & & & & & & 50 & 13 & 133 \\
\hline PT-46 & 26 & & 30 & & & & & & & 13 & 69 \\
\hline PT-47 & & 35 & & & & & & & 52 & & 87 \\
\hline BBC-12 & & & & & 35 & & & & & & 35 \\
\hline BBC-14 & & & & & 35 & & & & & & 35 \\
\hline BBC-15 & & & & & 35 & 16 & & & & & 51 \\
\hline BHC-16 & & & & & 35 & & & & & & 35 \\
\hline BHC-17 & & & & & 25 & & & & 19 & & 44 \\
\hline BHC-18 & & & & & 35 & 16 & & & & & 51 \\
\hline
\end{tabular}




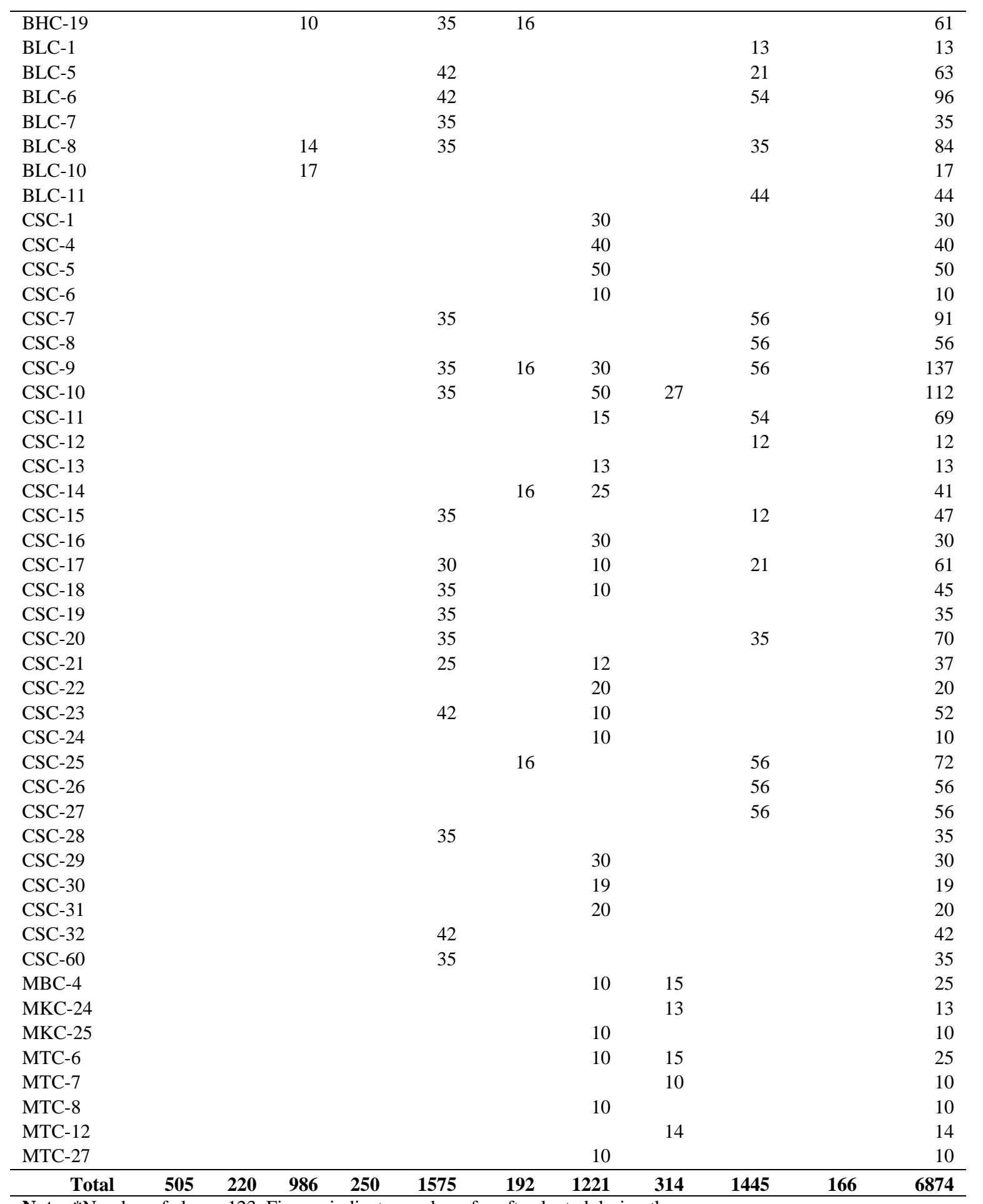

Note: *Number of clones 123. Figures indicate number of grafts planted during the year.

\section{Method of study of leaf flushing behaviour}

Surveyed TSO, Behrai, Seoni, for three years (2002-2004), to study leaf flushing behaviour of 123 teak clones of MP origin planted in 10 plots during the year 1977-1985. This study was carried out for three years (2002-2004) during the month of May-June (maximum temperature $40-46^{\circ} \mathrm{C}$ ) when the leaf flushing in teak of Madhya Pradesh occurs (Figs. 1 and 2). The leaf flushing period in MP teak clones planted in different plots of TSO, were recorded regularly as early (16-31 May), mid (1-15 June) and late (16-30 June), based on the period of leaf flushing, and the indices and codes used for leaf flushing are mentioned (Table 4).

Table 4. Leaf flushing indices and codes.

\begin{tabular}{llll}
\hline Category & Indices & Code & Period of leaf flushing \\
\hline Early & E & 1 & May 16 - May 31 \\
Mid & M & 2 & June 01 - June 15 \\
Late & L & 3 & June 16 - June 30 \\
\hline
\end{tabular}




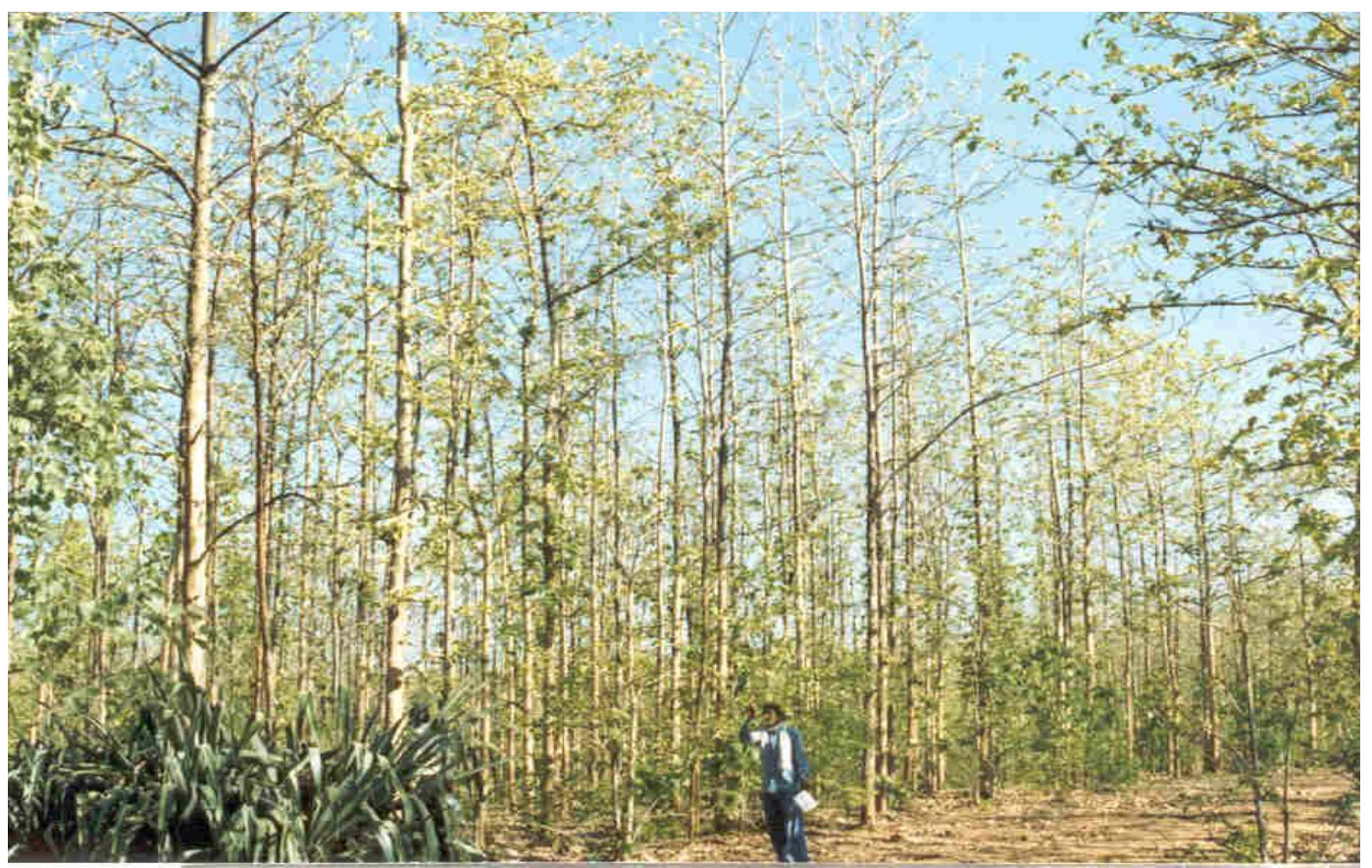

Figure 1. Leaf flushing in teak forest of Madhya Pradesh during the month of June.

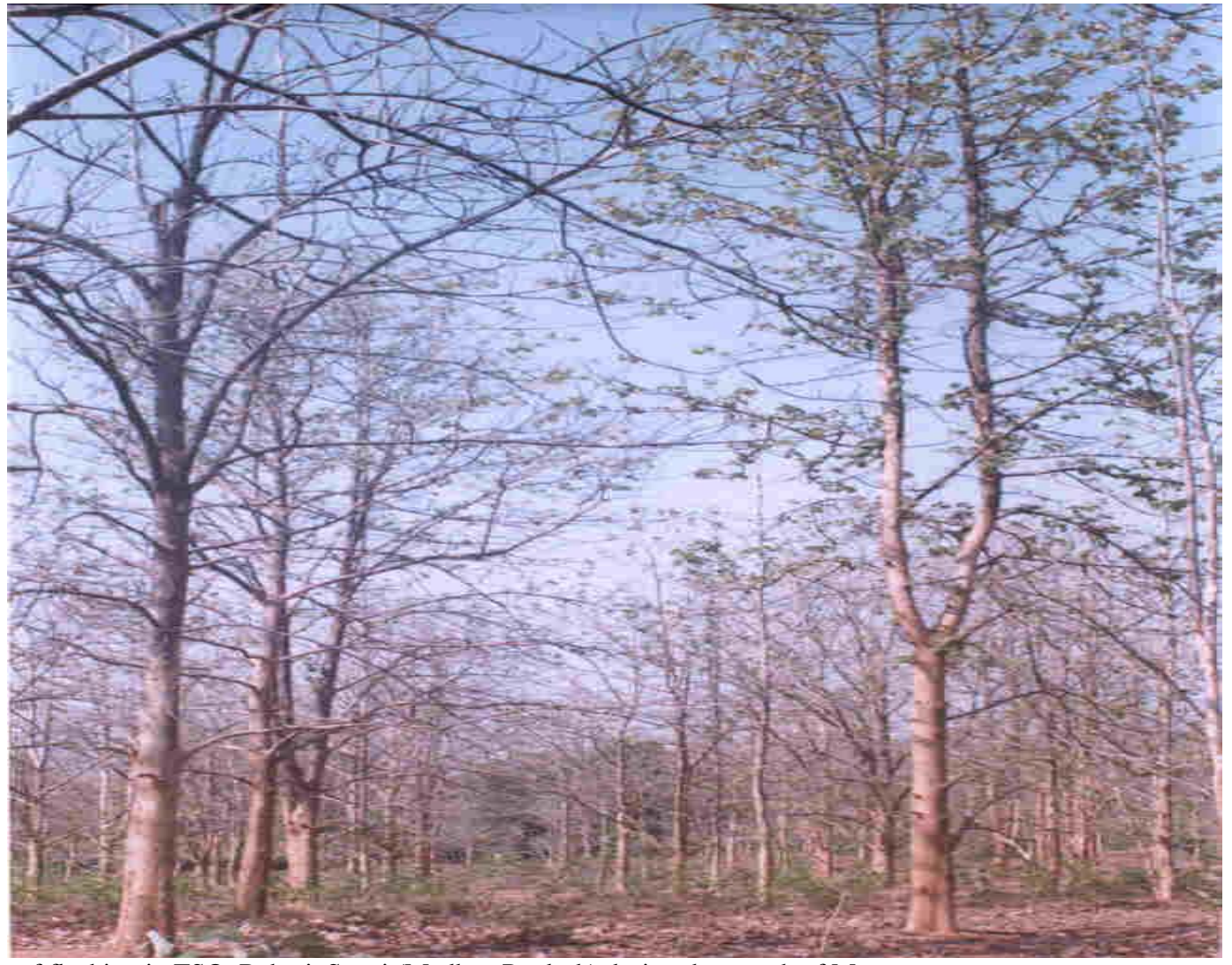

Figure 2. Leaf flushing in TSO, Behrai, Seoni (Madhya Pradesh) during the month of May.

To record the observations on the leaf flushing periods of teak clones, each and every plot of TSO, Behrai, Seoni, was visited four times, such as $15^{\text {th }}$ May, $31^{\text {st }}$ May, $15^{\text {th }}$ June and $30^{\text {th }}$ June, in a year during the period of leaf flushing in teak of Madhya Pradesh. An individual ramet of a clone was attended to record leaf flushing. Observations were recorded for all the clones separately minimum for 10 ramets of a clone in a plot and the leaf flushing period of each clone was worked out separately on the basis of average observations in different plots and also pooled mean of three years observations. The observations on leaf flushing periods were recorded by using numerical figures, such as 1,2 and 3 as code for E (early), M (mid) and L (late) respectively (Table 5) for statistical analysis. The pooled data on leaf flushing period, within and over the years were again divided into three groups based on the mean values, such as early 
$(<1.50)$, mid $(>1.50-<2.50)$ and late $(>2.50)$ and then subjected to ANOVA (RBD) tests (Gomez \& Gomez, 1984).

Table 5. Observations on leaf flushing behaviour of 19 MP teak clones planted during the year 1977 in TSO, Behrai, Seoni (Plot I).

\begin{tabular}{|c|c|c|c|c|c|}
\hline \multirow{3}{*}{$\begin{array}{l}\text { MP } \\
\text { teak clone }\end{array}$} & \multicolumn{5}{|c|}{ Leaf flushing } \\
\hline & \multicolumn{4}{|c|}{ Code* } & \multirow{2}{*}{ Period** } \\
\hline & 2002 & 2003 & 2004 & Mean & \\
\hline B-1 & 2.00 & 2.00 & 2.00 & 2.00 & $\bar{M}$ \\
\hline $\mathrm{C}-1$ & 3.00 & 2.00 & 2.00 & 2.33 & M \\
\hline $\mathrm{C}-2$ & 2.00 & 1.00 & 1.00 & 1.33 & $\mathrm{E}$ \\
\hline $\mathrm{C}-3$ & 2.00 & 1.00 & 1.00 & 1.33 & $\mathrm{E}$ \\
\hline C-6 & 2.00 & 2.00 & 2.00 & 2.00 & M \\
\hline C-8 & 1.00 & 2.00 & 1.00 & 1.33 & $\mathrm{E}$ \\
\hline C-9 & 1.00 & 1.00 & 1.00 & 1.00 & $\mathrm{E}$ \\
\hline $\mathrm{C}-10$ & 2.00 & 3.00 & 2.00 & 2.33 & M \\
\hline $\mathrm{C}-11$ & 2.00 & 1.00 & 1.00 & 1.33 & $\mathrm{E}$ \\
\hline $\mathrm{C}-12$ & 2.00 & 2.00 & 2.00 & 2.00 & M \\
\hline F-1 & 1.00 & 1.00 & 1.00 & 1.00 & $\mathrm{E}$ \\
\hline G-1 & 1.00 & 1.00 & 1.00 & 1.00 & $\mathrm{E}$ \\
\hline K-1 & 2.00 & 1.00 & 1.00 & 1.33 & $\mathrm{E}$ \\
\hline PT-1 & 2.00 & 1.00 & 1.00 & 1.33 & $\mathrm{E}$ \\
\hline PT-3 & 2.00 & 2.00 & 2.00 & 2.00 & M \\
\hline PT-26 & 2.00 & 1.00 & 1.00 & 1.33 & $\mathrm{E}$ \\
\hline PT-41 & 2.00 & 1.00 & 1.00 & 1.33 & $\mathrm{E}$ \\
\hline PT-45 & 2.00 & 1.00 & 1.00 & 1.33 & $\mathrm{E}$ \\
\hline PT-46 & 1.00 & 1.00 & 1.00 & 1.00 & $\mathrm{E}$ \\
\hline
\end{tabular}

\section{RESULTS}

The observations noticed during the course of study in plot-I, where 19 MP teak clones were planted during the year 1977 (Table 5), revealed that in the year 2002, 5 clones showed early leaf flushing during the period $16^{\text {th }}$ May-31 ${ }^{\text {st }}$ May, whereas 13 clones exhibited mid leaf flushing during the period $1^{\text {st }}$ June to $15^{\text {th }}$ June and only one clone revealed late leaf flushing during the period $16^{\text {th }}$ June to $30^{\text {th }}$ June. In 2003, it was observed that 12 clones showed early leaf flushing, 6 clones exhibited mid leaf flushing and only one clone showed late leaf flushing. In 2004, it was recorded that 13 clones as early and 6 clones as mid leaf flushers. The mean of three years observations revealed that out of 19 clones, 13 clones, viz. C-2, 3, 8, 9, 11, F-1, G-1, K-1 and PT-1, 26, 41, 45, 46 showed early leaf flushing, whereas 6 clones, viz. B-1, C-1, 6, 10, 12 and PT-3 observed as mid leaf flushers.

In plot-II, where 9 MP teak clones were planted during 1978 (Table 6), it was observed that in the year 2002, 4 clones exhibited early leaf flushing, whereas 4 clones were mid leaf flushers and only one clone as late leaf flusher. In 2003, the observations revealed that 5 clones were early leaf flushers and 4 clones were mid leaf flushers. In 2004, it was recorded that 7 clones showed early leaf flushing whereas 2 clones exhibited mid leaf flushing. The average of three years observations showed 5 clones, viz. C-4 and PT-1, 45, 46, 47 exhibited early leaf flushing followed by 4 clones, viz. C-3, 5, 11, 15 as mid leaf flushers.

Table 6. Observations on leaf flushing behaviour of 9 MP teak clones planted during the year 1978 in TSO, Behrai, Seoni (Plot II).

\begin{tabular}{lcccrr}
\hline \multirow{2}{*}{$\begin{array}{l}\text { MP } \\
\text { teak clone }\end{array}$} & \multicolumn{5}{c}{ Leaf flushing } \\
\cline { 2 - 5 } & $\mathbf{2 0 0 2}$ & $\mathbf{2 0 0 3}$ & $\mathbf{2 0 0 4}$ & Mean & Period** \\
\cline { 2 - 5 } C-3 & 2.00 & 2.00 & 1.00 & 1.67 & M \\
C-4 & 2.00 & 1.00 & 1.00 & 1.33 & $\mathrm{E}$ \\
C-5 & 3.00 & 2.00 & 2.00 & 2.33 & $\mathrm{M}$ \\
C-11 & 2.00 & 2.00 & 1.00 & 1.67 & $\mathrm{M}$ \\
C-15 & 2.00 & 2.00 & 2.00 & 2.00 & $\mathrm{M}$ \\
PT-1 & 1.00 & 1.00 & 1.00 & 1.00 & $\mathrm{E}$ \\
PT-45 & 1.00 & 1.00 & 1.00 & 1.00 & $\mathrm{E}$ \\
PT-46 & 1.00 & 1.00 & 1.00 & 1.00 & $\mathrm{E}$ \\
PT-47 & 1.00 & 1.00 & 1.00 & 1.00 & $\mathrm{E}$ \\
\hline Note: & & & & \\
\end{tabular}

Note: *= Observations recorded during the month of May-June; **=E and M indicate early and mid and leaf flushing respectively; Data based on 10 ramets. 
In plot-III, where 42 MP teak clones were planted during the year 1979 (I) (Table 7), it was recorded that only one clone showed early leaf flushing whereas 29 clones exhibited mid leaf flushing and 12 clones as late leaf flushers. In, 2003, the observations showed that 7 clones were early leaf flushers, 29 as mid and 6 clones as late leaf flushers. In, 2004, 9 clones showed early leaf flushing whereas $28 \mathrm{mid}$ and 5 clones exhibited late leaf flushing. The average of three years observations revealed that 7 clones, viz. C-2, 3, 8, 54, F-1, PT-46 and BHC-19 showed early leaf flushing, 28 clones, viz. C-5, 15, 17, 20, 22, 23, 24, 25, 26, 27, 28, 35, 37, 38, 39, 40, 42, 45, 46, 47, 48, 49, 50, 51, 53, 56, 57 and 60 as mid leaf flushers and 7 clones, viz. C-1, 19, 34, 41, 44 and BLC-8, 10 as late leaf flushers.

Table 7. Observations on leaf flushing behaviour of 42 M.P. teak clones planted during the year 1979 (I) in TSO, Behrai, Seoni (Plot III).

\begin{tabular}{|c|c|c|c|c|c|}
\hline \multirow{3}{*}{$\begin{array}{l}\text { MP } \\
\text { teak clone }\end{array}$} & \multicolumn{5}{|c|}{ Leaf flushing } \\
\hline & \multicolumn{4}{|c|}{ Code* } & \multirow{2}{*}{ Period $* *$} \\
\hline & 2002 & 2003 & 2004 & Mean & \\
\hline $\mathrm{C}-1$ & 3.00 & 2.00 & 3.00 & 2.67 & $\mathrm{~L}$ \\
\hline $\mathrm{C}-2$ & 2.00 & 1.00 & 1.00 & 1.33 & $\mathrm{E}$ \\
\hline $\mathrm{C}-3$ & 2.00 & 1.00 & 1.00 & 1.33 & $\mathrm{E}$ \\
\hline C-5 & 3.00 & 2.00 & 2.00 & 2.33 & M \\
\hline $\mathrm{C}-8$ & 2.00 & 1.00 & 1.00 & 1.33 & $\mathrm{E}$ \\
\hline $\mathrm{C}-5$ & 2.00 & 2.00 & 2.00 & 2.00 & M \\
\hline $\mathrm{C}-17$ & 2.00 & 2.00 & 2.00 & 2.00 & M \\
\hline C-19 & 3.00 & 3.00 & 2.00 & 2.67 & $\mathrm{~L}$ \\
\hline $\mathrm{C}-20$ & 2.00 & 2.00 & 2.00 & 2.00 & M \\
\hline $\mathrm{C}-22$ & 2.00 & 2.00 & 2.00 & 2.00 & M \\
\hline $\mathrm{C}-23$ & 2.00 & 2.00 & 2.00 & 2.00 & M \\
\hline C-24 & 2.00 & 2.00 & 3.00 & 2.33 & M \\
\hline $\mathrm{C}-25$ & 3.00 & 2.00 & 2.00 & 2.33 & M \\
\hline C-26 & 2.00 & 2.00 & 2.00 & 2.00 & M \\
\hline $\mathrm{C}-27$ & 2.00 & 2.00 & 2.00 & 2.00 & M \\
\hline $\mathrm{C}-28$ & 2.00 & 2.00 & 2.00 & 2.00 & M \\
\hline $\mathrm{C}-34$ & 3.00 & 3.00 & 2.00 & 2.67 & $\mathrm{~L}$ \\
\hline $\mathrm{C}-35$ & 3.00 & 2.00 & 2.00 & 2.33 & M \\
\hline $\mathrm{C}-37$ & 2.00 & 2.00 & 2.00 & 2.00 & M \\
\hline $\mathrm{C}-38$ & 2.00 & 2.00 & 2.00 & 2.00 & M \\
\hline C-39 & 2.00 & 2.00 & 2.00 & 2.00 & M \\
\hline$C-40$ & 2.00 & 2.00 & 1.00 & 1.67 & M \\
\hline C-41 & 3.00 & 3.00 & 3.00 & 3.00 & $\mathrm{~L}$ \\
\hline C- -42 & 3.00 & 2.00 & 2.00 & 2.33 & M \\
\hline C-44 & 3.00 & 3.00 & 3.00 & 3.00 & $\mathrm{~L}$ \\
\hline$C-45$ & 2.00 & 2.00 & 2.00 & 2.00 & M \\
\hline C-46 & 2.00 & 2.00 & 2.00 & 2.00 & M \\
\hline C-47 & 2.00 & 2.00 & 2.00 & 2.00 & M \\
\hline C- -48 & 2.00 & 2.00 & 2.00 & 2.00 & M \\
\hline C- -49 & 2.00 & 2.00 & 2.00 & 2.00 & M \\
\hline $\mathrm{C}-50$ & 2.00 & 2.00 & 2.00 & 2.00 & M \\
\hline C-51 & 2.00 & 2.00 & 2.00 & 2.00 & M \\
\hline C-53 & 2.00 & 2.00 & 2.00 & 2.00 & M \\
\hline C-54 & 2.00 & 1.00 & 1.00 & 1.33 & $\mathrm{E}$ \\
\hline C-56 & 3.00 & 2.00 & 2.00 & 2.33 & M \\
\hline C-57 & 2.00 & 2.00 & 2.00 & 2.00 & M \\
\hline$C-60$ & 2.00 & 2.00 & 1.00 & 1.67 & M \\
\hline F-1 & 1.00 & 1.00 & 1.00 & 1.00 & $\mathrm{E}$ \\
\hline PT-46 & 2.00 & 1.00 & 1.00 & 1.33 & $\mathrm{E}$ \\
\hline BHC-19 & 2.00 & 1.00 & 1.00 & 1.33 & $\mathrm{E}$ \\
\hline BLC- 8 & 3.00 & 3.00 & 2.00 & 2.67 & $\mathrm{~L}$ \\
\hline BLC-10 & 3.00 & 3.00 & 3.00 & 3.00 & $\mathrm{~L}$ \\
\hline
\end{tabular}

In plot IV, where 16 MP teak clones were planted during the year 1979 (II) (Table 8), it was noticed that in the year 2002, 12 clones showed mid leaf flushing and 4 clones exhibited late leaf flushing. In 2003, 2 clones showed early leaf flushing, 12 clones as mid and 2 clones as late leaf flushers. In 2004, 2 clones exhibited early leaf flushing, 12 as mid and 2 clones showed late leaf flushing. The average of three years observations exhibited that only one clone, viz. C-54 
as early, 13 clones, viz. C-8, 22, 25, 26, 27, 28, 35, 38, 39, 40, 45, 46, 52 as mid and 2 clones, viz. C-44, 56 as late leaf flushers.

Table 8. Observations on leaf flushing behaviour of 16 MP teak clones planted during the year 1979 (II) in TSO, Behrai, Seoni (Plot IV).

\begin{tabular}{|c|c|c|c|c|c|}
\hline \multirow{3}{*}{$\begin{array}{l}\text { MP } \\
\text { teak clone }\end{array}$} & \multicolumn{5}{|c|}{ Leaf flushing } \\
\hline & \multicolumn{4}{|c|}{ Code* } & \multirow{2}{*}{ Period $* *$} \\
\hline & 2002 & 2003 & 2004 & Mean & \\
\hline C-8 & 2.00 & 1.00 & 2.00 & 1.67 & M \\
\hline C- 22 & 2.00 & 2.00 & 2.00 & 2.00 & $\mathrm{M}$ \\
\hline C-25 & 2.00 & 2.00 & 3.00 & 2.33 & M \\
\hline C-26 & 2.00 & 2.00 & 2.00 & 2.00 & M \\
\hline C-27 & 2.00 & 2.00 & 2.00 & 2.00 & $\mathrm{M}$ \\
\hline C-28 & 3.00 & 2.00 & 2.00 & 2.33 & M \\
\hline $\mathrm{C}-35$ & 2.00 & 2.00 & 2.00 & 2.00 & M \\
\hline C-38 & 2.00 & 2.00 & 2.00 & 2.00 & M \\
\hline C-39 & 2.00 & 2.00 & 2.00 & 2.00 & M \\
\hline C-40 & 2.00 & 2.00 & 2.00 & 2.00 & M \\
\hline C-44 & 3.00 & 3.00 & 3.00 & 3.00 & $\mathrm{~L}$ \\
\hline C-45 & 2.00 & 2.00 & 1.00 & 1.67 & M \\
\hline C-46 & 2.00 & 2.00 & 2.00 & 2.00 & M \\
\hline C-52 & 3.00 & 2.00 & 2.00 & 2.33 & M \\
\hline C-54 & 2.00 & 1.00 & 1.00 & 1.33 & E \\
\hline C-56 & 3.00 & 3.00 & 2.00 & 2.67 & L \\
\hline
\end{tabular}

Note: $*=$ Observations recorded during the month of May-June; $* *=\mathrm{E}, \mathrm{M}$ and $\mathrm{L}$ indicate early, mid and late leaf flushing respectively; Data based on 10 ramets.

In plot V, where 46 MP teak clones were planted during the year 1980 (I) (Table 9), it was recorded that in the year 2002, 4 clones showed early leaf flushing, 22 clones mid and 20 clones exhibited late leaf flushing. In 2003,7 clones showed early leaf flushing, 18 clones mid and 21 clones late leaf flushing. In 2004, 9 clones exhibited early leaf flushing, $29 \mathrm{mid}$ and 8 clones as late leaf flushers. The average of three years observations exhibited that 8 clones, viz. C-4, 8, 9, F-1, K-1, PT-1, BHC-19 and CSC-9 as early, 22 clones, viz. C-10, 11, 27, 38, 39, 40, 45, 57, 60, M-1, BBC12, 15, BHC-16, BLC-5 and CSC-15, 18, 19, 20, 21, 23, 32, 60 as mid and 16 clones, viz. C-25, 26, 41, 49, 50, 59, BBC-14, BHC-17, 18, BLC-6, 7, 8 and CSC-7, 10, 17, 28 as late leaf flushers.

Table 9. Observations on leaf flushing behaviour of $46 \mathrm{MP}$ teak clones planted during the year 1980 (I) in, TSO, Behrai, Seoni (Plot V).

\begin{tabular}{|c|c|c|c|c|c|}
\hline \multirow{3}{*}{$\begin{array}{l}\text { MP } \\
\text { teak clone }\end{array}$} & \multicolumn{5}{|c|}{ Leaf flushing } \\
\hline & \multicolumn{4}{|c|}{ Code* $^{*}$} & \multirow{2}{*}{ Period** } \\
\hline & 2002 & 2003 & 2004 & Mean & \\
\hline C-4 & 2.00 & 1.00 & 1.00 & 1.33 & $\mathrm{E}$ \\
\hline C-8 & 1.00 & 2.00 & 1.00 & 1.33 & $\mathrm{E}$ \\
\hline C-9 & 2.00 & 1.00 & 1.00 & 1.33 & E \\
\hline $\mathrm{C}-10$ & 2.00 & 3.00 & 2.00 & 2.33 & M \\
\hline $\mathrm{C}-11$ & 2.00 & 2.00 & 1.00 & 1.67 & M \\
\hline $\mathrm{C}-25$ & 3.00 & 3.00 & 2.00 & 2.67 & $\mathrm{~L}$ \\
\hline $\mathrm{C}-26$ & 2.00 & 3.00 & 3.00 & 2.67 & $\mathrm{~L}$ \\
\hline $\mathrm{C}-27$ & 2.00 & 2.00 & 2.00 & 2.00 & M \\
\hline $\mathrm{C}-38$ & 2.00 & 2.00 & 2.00 & 2.00 & M \\
\hline C-39 & 2.00 & 3.00 & 2.00 & 2.33 & M \\
\hline $\mathrm{C}-40$ & 2.00 & 3.00 & 2.00 & 2.33 & M \\
\hline $\mathrm{C}-41$ & 3.00 & 3.00 & 3.00 & 3.00 & $\mathrm{~L}$ \\
\hline$C-45$ & 2.00 & 2.00 & 2.00 & 2.00 & M \\
\hline C-49 & 2.00 & 3.00 & 3.00 & 2.67 & $\mathrm{~L}$ \\
\hline $\mathrm{C}-50$ & 3.00 & 3.00 & 2.00 & 2.67 & $\mathrm{~L}$ \\
\hline$C-57$ & 2.00 & 2.00 & 2.00 & 2.00 & M \\
\hline C-59 & 3.00 & 3.00 & 3.00 & 3.00 & $\mathrm{~L}$ \\
\hline$C-60$ & 3.00 & 2.00 & 2.00 & 2.33 & M \\
\hline $\mathrm{F}-1$ & 1.00 & 1.00 & 1.00 & 1.00 & $\mathrm{E}$ \\
\hline K-1 & 2.00 & 1.00 & 1.00 & 1.33 & $\mathrm{E}$ \\
\hline M-1 & 3.00 & 2.00 & 2.00 & 2.33 & M \\
\hline PT-1 & 1.00 & 1.00 & 1.00 & 1.00 & $\mathrm{E}$ \\
\hline BBC-12 & 2.00 & 2.00 & 2.00 & 2.00 & M \\
\hline BBC-14 & 3.00 & 3.00 & 2.00 & 2.67 & $\mathrm{~L}$ \\
\hline
\end{tabular}




\begin{tabular}{lllllc}
\hline BBC-15 & 2.00 & 2.00 & 2.00 & 2.00 & $\mathrm{M}$ \\
BHC-16 & 3.00 & 2.00 & 2.00 & 2.33 & $\mathrm{M}$ \\
BHC-17 & 3.00 & 3.00 & 2.00 & 2.67 & $\mathrm{~L}$ \\
BHC-18 & 3.00 & 3.00 & 2.00 & 2.67 & $\mathrm{~L}$ \\
BHC-19 & 1.00 & 1.00 & 1.00 & 1.00 & $\mathrm{E}$ \\
BLC-5 & 3.00 & 2.00 & 2.00 & 2.33 & $\mathrm{M}$ \\
BLC-6 & 3.00 & 3.00 & 3.00 & 3.00 & $\mathrm{~L}$ \\
BLC-7 & 3.00 & 3.00 & 3.00 & 3.00 & $\mathrm{~L}$ \\
BLC-8 & 2.00 & 3.00 & 3.00 & 2.67 & $\mathrm{~L}$ \\
CSC-7 & 3.00 & 3.00 & 3.00 & 3.00 & $\mathrm{~L}$ \\
CSC-9 & 2.00 & 1.00 & 1.00 & 1.33 & $\mathrm{E}$ \\
CSC-10 & 3.00 & 3.00 & 2.00 & 2.67 & $\mathrm{~L}$ \\
CSC-15 & 2.00 & 3.00 & 2.00 & 2.33 & $\mathrm{M}$ \\
CSC-17 & 3.00 & 3.00 & 2.00 & 2.67 & $\mathrm{~L}$ \\
CSC-18 & 2.00 & 2.00 & 2.00 & 2.00 & $\mathrm{M}$ \\
CSC-19 & 3.00 & 2.00 & 2.00 & 2.33 & $\mathrm{M}$ \\
CSC-20 & 3.00 & 2.00 & 2.00 & 2.33 & $\mathrm{M}$ \\
CSC-21 & 2.00 & 2.00 & 2.00 & 2.00 & $\mathrm{M}$ \\
CSC-23 & 2.00 & 2.00 & 2.00 & 2.00 & $\mathrm{M}$ \\
CSC-28 & 3.00 & 3.00 & 2.00 & 2.67 & $\mathrm{~L}$ \\
CSC-32 & 3.00 & 2.00 & 2.00 & 2.33 & $\mathrm{M}$ \\
CSC-60 & 2.00 & 3.00 & 2.00 & 2.33 & $\mathrm{M}$ \\
\hline Note: $*$ Observations recorded during the month of May-June; ** E, M and L \\
indicate early, mid and late leaf flushing respectively; Data based on 10 ramets.
\end{tabular}

In plot VI, where 12 MP teak clones were planted during the year 1980 (II) (Table 10), it was observed that in the year 2002, 3 clones showed early leaf flushing, 4 clones as mid and 5 clones as late leaf flushers. In 2003, 3 clones recorded as early, 7 clones as mid and 2 clones as late leaf flushers. In 2004, 3 clones showed early leaf flushing and 9 clones as mid leaf flushers. The average of three years observations revealed that 3 clones, viz. PT-1, BHC-19 and CSC9 as early leaf flushers, 7 clones viz. C-28, 29, 32, 35, 45, BBC-15 and CSC-25 as mid and 2 clones, viz. BHC-18 and CSC-14 as late leaf flushers.

Table 10. Observations on leaf flushing behaviour of 12 MP teak clones planted during the year 1980 (II) in TSO, Behrai, Seoni (Plot VI).

\begin{tabular}{|c|c|c|c|c|c|}
\hline \multirow{3}{*}{$\begin{array}{l}\text { MP } \\
\text { teak clone }\end{array}$} & \multicolumn{5}{|c|}{ Leaf flushing } \\
\hline & \multicolumn{4}{|c|}{ Code* } & \multirow{2}{*}{ Period $* *$} \\
\hline & 2002 & 2003 & 2004 & Mean & \\
\hline $\mathrm{C}-28$ & 2.00 & 2.00 & 2.00 & 2.00 & $\bar{M}$ \\
\hline C-29 & 3.00 & 2.00 & 2.00 & 2.33 & M \\
\hline $\mathrm{C}-32$ & 3.00 & 2.00 & 2.00 & 2.33 & M \\
\hline $\mathrm{C}-35$ & 2.00 & 2.00 & 2.00 & 2.00 & M \\
\hline$C-45$ & 2.00 & 2.00 & 2.00 & 2.00 & M \\
\hline PT-1 & 1.00 & 1.00 & 1.00 & 1.00 & $\mathrm{E}$ \\
\hline BBC-15 & 3.00 & 2.00 & 2.00 & 2.33 & M \\
\hline BHC-18 & 3.00 & 3.00 & 2.00 & 2.67 & $\mathrm{~L}$ \\
\hline BHC-19 & 1.00 & 1.00 & 1.00 & 1.00 & $\mathrm{E}$ \\
\hline CSC-9 & 1.00 & 1.00 & 1.00 & 1.00 & $\mathrm{E}$ \\
\hline CSC-14 & 3.00 & 3.00 & 2.00 & 2.67 & $\mathrm{~L}$ \\
\hline CSC-25 & 2.00 & 2.00 & 2.00 & 2.00 & M \\
\hline
\end{tabular}

Note: * Observations recorded during the month of May-June; **=E, M and L indicate early, mid and late leaf flushing respectively; Data based on 10 ramets.

Table 11. Observations on leaf flushing behaviour of 48 MP teak clones planted during the year 1981 (I) in TSO, Behrai, Seoni (Plot VII).

\begin{tabular}{lcccrr}
\hline \multirow{2}{*}{$\begin{array}{l}\text { MP } \\
\text { teak clone }\end{array}$} & \multicolumn{5}{c}{ Leaf flushing } \\
\cline { 2 - 5 } & $\mathbf{2 0 0 2}$ & $\mathbf{2 0 0 3}$ & $\mathbf{2 0 0 4}$ & Mean & \multirow{2}{*}{ Period** } \\
\cline { 2 - 5 } C-1 & 3.00 & 3.00 & 2.00 & 2.67 & $\mathrm{~L}$ \\
C-2 & 2.00 & 2.00 & 1.00 & 1.67 & $\mathrm{M}$ \\
C-4 & 2.00 & 1.00 & 2.00 & 1.67 & $\mathrm{M}$ \\
C-5 & 3.00 & 2.00 & 2.00 & 2.33 & $\mathrm{M}$ \\
C-6 & 2.00 & 3.00 & 2.00 & 2.33 & $\mathrm{M}$ \\
C-18 & 2.00 & 2.00 & 2.00 & 2.00 & $\mathrm{M}$ \\
\hline
\end{tabular}




\begin{tabular}{|c|c|c|c|c|c|}
\hline C-19 & 2.00 & 3.00 & 3.00 & 2.67 & $\mathrm{~L}$ \\
\hline $\mathrm{C}-25$ & 3.00 & 3.00 & 3.00 & 3.00 & $\mathrm{~L}$ \\
\hline $\mathrm{C}-26$ & 3.00 & 3.00 & 2.00 & 2.67 & $\mathrm{~L}$ \\
\hline C-34 & 3.00 & 2.00 & 3.00 & 2.67 & $\mathrm{~L}$ \\
\hline$C-36$ & 3.00 & 2.00 & 2.00 & 2.33 & M \\
\hline $\mathrm{C}-40$ & 2.00 & 2.00 & 1.00 & 1.67 & M \\
\hline C-41 & 3.00 & 3.00 & 3.00 & 3.00 & $\mathrm{~L}$ \\
\hline C-42 & 3.00 & 3.00 & 3.00 & 3.00 & $\mathrm{~L}$ \\
\hline$C-45$ & 2.00 & 2.00 & 2.00 & 2.00 & M \\
\hline C-47 & 3.00 & 2.00 & 2.00 & 2.33 & M \\
\hline C- 48 & 2.00 & 3.00 & 2.00 & 2.33 & M \\
\hline C-49 & 3.00 & 3.00 & 2.00 & 2.67 & $\mathrm{~L}$ \\
\hline C-56 & 3.00 & 3.00 & 3.00 & 3.00 & $\mathrm{~L}$ \\
\hline C-57 & 2.00 & 3.00 & 3.00 & 2.67 & $\mathrm{~L}$ \\
\hline PT-1 & 1.00 & 1.00 & 1.00 & 1.00 & $\mathrm{E}$ \\
\hline PT-5 & 2.00 & 3.00 & 2.00 & 2.33 & M \\
\hline PT-6 & 3.00 & 2.00 & 2.00 & 2.33 & M \\
\hline PT-7 & 3.00 & 3.00 & 3.00 & 3.00 & $\mathrm{~L}$ \\
\hline CSC-1 & 3.00 & 2.00 & 2.00 & 2.33 & M \\
\hline CSC-4 & 3.00 & 2.00 & 2.00 & 2.33 & M \\
\hline CSC-5 & 2.00 & 2.00 & 2.00 & 2.00 & M \\
\hline CSC-6 & 2.00 & 2.00 & 2.00 & 2.00 & M \\
\hline CSC-9 & 1.00 & 1.00 & 1.00 & 1.00 & $\mathrm{E}$ \\
\hline CSC-10 & 2.00 & 3.00 & 3.00 & 2.67 & $\mathrm{~L}$ \\
\hline CSC-11 & 3.00 & 3.00 & 3.00 & 3.00 & $\mathrm{~L}$ \\
\hline CSC-13 & 3.00 & 3.00 & 2.00 & 2.67 & $\mathrm{~L}$ \\
\hline CSC-14 & 3.00 & 3.00 & 2.00 & 2.67 & $\mathrm{~L}$ \\
\hline CSC-16 & 3.00 & 3.00 & 2.00 & 2.67 & $\mathrm{~L}$ \\
\hline CSC-17 & 3.00 & 3.00 & 2.00 & 2.67 & $\mathrm{~L}$ \\
\hline CSC-18 & 2.00 & 2.00 & 2.00 & 2.00 & M \\
\hline CSC-21 & 3.00 & 2.00 & 2.00 & 2.33 & M \\
\hline CSC-22 & 2.00 & 2.00 & 2.00 & 2.00 & M \\
\hline CSC-23 & 2.00 & 2.00 & 2.00 & 2.00 & M \\
\hline CSC-24 & 3.00 & 2.00 & 2.00 & 2.33 & M \\
\hline CSC-29 & 2.00 & 2.00 & 3.00 & 2.33 & M \\
\hline CSC-30 & 3.00 & 2.00 & 2.00 & 2.33 & M \\
\hline CSC-31 & 3.00 & 2.00 & 2.00 & 2.33 & M \\
\hline MBC-4 & 2.00 & 2.00 & 2.00 & 2.00 & M \\
\hline MKC-25 & 3.00 & 2.00 & 2.00 & 2.33 & M \\
\hline MTC-6 & 2.00 & 2.00 & 2.00 & 2.00 & M \\
\hline MTC-8 & 2.00 & 2.00 & 3.00 & 2.33 & M \\
\hline MTC-27 & 3.00 & 2.00 & 2.00 & 2.33 & M \\
\hline
\end{tabular}

Note: $*=$ Observations recorded during the month of May-June; $* *=\mathrm{E}, \mathrm{M}$ and $\mathrm{L}$ indicate early, mid and late leaf flushing respectively; Data based on 10 ramets.

In plot VII, where 48 MP teak clones were planted during the year 1981 (I) (Table 11), it was recorded that in the year 2002, 2 clones showed early leaf flushing, 20 clones exhibited mid leaf flushing and 26 showed late leaf flushing. In 2003, 3 clones showed early leaf flushing, 26 as mid and 19 clones as late leaf flushers. In 2004, 4 clones were recorded as early leaf flushers, 32 clones as mid and 12 clones as late leaf flushers. The average of three years observations revealed that 2 clones, viz. PT-1 and CSC-9 as early leaf flushers, 29 clones, viz. C-2, 4, 5, 6, 18, 36, 40, 45, 47, 48, PT-5, 6, CSC-1, 4, 5, 6, 18, 21, 22, 23, 24, 29, 30, 31, MBC-4, MKC-25 and MTC-6, 8, 27 as mid leaf flushers, 17 clones, viz. C-1, 19, 25, 26, 34, 41, 42, 49, 56, 57, PT-7 and CSC-10, 11, 13, 14, 16, 17 as late leaf flushers.

Table 12. Observations on leaf flushing behaviour of $15 \mathrm{MP}$ teak clones planted during the year 1981 (II) in Behrai, Seoni (Plot VIII).

\begin{tabular}{|c|c|c|c|c|c|}
\hline \multirow{3}{*}{$\begin{array}{l}\text { MP } \\
\text { teak Clone }\end{array}$} & \multicolumn{5}{|c|}{ Leaf flushing } \\
\hline & \multicolumn{4}{|c|}{ Code $^{*}$} & \multirow{2}{*}{ Period** } \\
\hline & 2002 & 2003 & 2004 & Mean & \\
\hline $\mathrm{C}-1$ & 3.00 & 3.00 & 2.00 & 2.67 & $\mathrm{~L}$ \\
\hline C-4 & 2.00 & 1.00 & 1.00 & 1.33 & E \\
\hline C-31 & 2.00 & 2.00 & 2.00 & 2.00 & M \\
\hline $\mathrm{C}-34$ & 3.00 & 3.00 & 2.00 & 2.67 & $\mathrm{~L}$ \\
\hline C-42 & 2.00 & 2.00 & 2.00 & 2.00 & $\mathrm{M}$ \\
\hline
\end{tabular}




\begin{tabular}{lllllr}
\hline C-45 & 2.00 & 2.00 & 1.00 & 1.67 & $\mathrm{M}$ \\
C-47 & 3.00 & 2.00 & 3.00 & 2.67 & $\mathrm{~L}$ \\
PT-1 & 1.00 & 1.00 & 1.00 & 1.00 & $\mathrm{E}$ \\
PT-7 & 3.00 & 2.00 & 2.00 & 2.33 & $\mathrm{M}$ \\
CSC-10 & 3.00 & 2.00 & 2.00 & 2.33 & $\mathrm{M}$ \\
MBC-4 & 2.00 & 2.00 & 2.00 & 2.00 & $\mathrm{M}$ \\
MKC-24 & 2.00 & 2.00 & 2.00 & 2.00 & $\mathrm{M}$ \\
MTC-6 & 2.00 & 2.00 & 3.00 & 2.33 & $\mathrm{M}$ \\
MTC-7 & 2.00 & 2.00 & 2.00 & 2.00 & $\mathrm{M}$ \\
MTC-12 & 3.00 & 2.00 & 2.00 & 2.33 & $\mathrm{M}$ \\
\hline Note: * Observations recorded during the
\end{tabular}

Note: *= Observations recorded during the month of May-June; **=E, M and L indicate early, mid and late leaf flushing respectively; Data based on 10 ramets.

In plot VIII, where 15 MP teak clones were planted during the year 1981 (II) (Table 12), it was noticed that in the year 2002, only one clone showed early leaf flushing, 8 clones exhibited mid and 6 clones revealed late leaf flushing. In 2003, 2 clones showed early leaf flushing, 11 mid leaf flushing and two clones as late leaf flushers. In 2004, 3 clones showed as early leaf flushers, 10 clones as mid and 2 clones as late leaf flushers. The average of three years observations revealed that two clones, viz. C-4 and PT-1 as early, 10 clones, viz. C-31, 42, 45, PT-7, CSC-10, MBC-4, MKC-24 and MTC-6, 7, 12 as mid and 3 clones, viz. C-1, 34, 47 as late leaf flushers.

Table 13. Observations on leaf flushing behaviour of 37 MP teak clones plant during the year 1982 in TSO, Behrai, Seoni (Plot IX).

\begin{tabular}{|c|c|c|c|c|c|}
\hline \multirow{3}{*}{$\begin{array}{l}\text { MP } \\
\text { teak clone }\end{array}$} & \multicolumn{5}{|c|}{ Leaf flushing } \\
\hline & \multicolumn{4}{|c|}{ Code* } & \multirow{2}{*}{ Period $* *$} \\
\hline & 2002 & 2003 & 2004 & Mean & \\
\hline C-6 & 3.00 & 2.00 & 2.00 & 2.33 & $\bar{M}$ \\
\hline $\mathrm{C}-7$ & 2.00 & 2.00 & 3.00 & 2.33 & M \\
\hline C-8 & 2.00 & 2.00 & 2.00 & 2.00 & M \\
\hline C-9 & 2.00 & 2.00 & 1.00 & 1.67 & M \\
\hline $\mathrm{C}-10$ & 3.00 & 2.00 & 2.00 & 2.33 & $\mathrm{M}$ \\
\hline $\mathrm{C}-11$ & 1.00 & 1.00 & 1.00 & 1.00 & $\mathrm{E}$ \\
\hline C-12 & 3.00 & 2.00 & 2.00 & 2.33 & M \\
\hline C-14 & 2.00 & 3.00 & 2.00 & 2.33 & $\mathrm{M}$ \\
\hline C- 15 & 2.00 & 3.00 & 3.00 & 2.67 & $\mathrm{~L}$ \\
\hline C-16 & 2.00 & 2.00 & 2.00 & 2.00 & M \\
\hline $\mathrm{C}-17$ & 2.00 & 2.00 & 2.00 & 2.00 & M \\
\hline C-18 & 3.00 & 2.00 & 3.00 & 2.67 & $\mathrm{~L}$ \\
\hline $\mathrm{C}-26$ & 3.00 & 2.00 & 2.00 & 2.33 & M \\
\hline $\mathrm{C}-30$ & 3.00 & 2.00 & 2.00 & 2.33 & M \\
\hline C-33 & 2.00 & 2.00 & 2.00 & 2.00 & M \\
\hline C-42 & 3.00 & 3.00 & 3.00 & 3.00 & $\mathrm{~L}$ \\
\hline C-59 & 3.00 & 3.00 & 3.00 & 3.00 & $\mathrm{~L}$ \\
\hline PT-3 & 3.00 & 3.00 & 2.00 & 2.67 & $\mathrm{~L}$ \\
\hline PT-45 & 1.00 & 1.00 & 1.00 & 1.00 & $\mathrm{E}$ \\
\hline PT-47 & 2.00 & 1.00 & 1.00 & 1.33 & $\mathrm{E}$ \\
\hline BHC-17 & 2.00 & 2.00 & 2.00 & 2.00 & $\mathrm{M}$ \\
\hline BLC-1 & 3.00 & 3.00 & 2.00 & 2.67 & $\mathrm{~L}$ \\
\hline BLC-5 & 3.00 & 3.00 & 2.00 & 2.67 & $\mathrm{~L}$ \\
\hline BLC-6 & 3.00 & 3.00 & 2.00 & 2.67 & $\mathrm{~L}$ \\
\hline BLC-8 & 3.00 & 2.00 & 2.00 & 2.33 & M \\
\hline BLC-11 & 2.00 & 2.00 & 2.00 & 2.00 & M \\
\hline CSC-7 & 3.00 & 2.00 & 2.00 & 2.33 & M \\
\hline CSC-8 & 3.00 & 2.00 & 2.00 & 2.33 & M \\
\hline CSC-9 & 2.00 & 2.00 & 1.00 & 1.67 & M \\
\hline CSC-11 & 3.00 & 2.00 & 2.00 & 2.33 & $\mathrm{M}$ \\
\hline CSC-12 & 3.00 & 2.00 & 2.00 & 2.33 & M \\
\hline CSC-15 & 3.00 & 3.00 & 3.00 & 3.00 & $\mathrm{~L}$ \\
\hline CSC-17 & 3.00 & 3.00 & 2.00 & 2.67 & $\mathrm{~L}$ \\
\hline CSC-20 & 3.00 & 3.00 & 2.00 & 2.67 & $\mathrm{~L}$ \\
\hline CSC-25 & 3.00 & 3.00 & 2.00 & 2.67 & $\mathrm{~L}$ \\
\hline CSC-26 & 2.00 & 2.00 & 2.00 & 2.00 & M \\
\hline CSC-27 & 3.00 & 2.00 & 2.00 & 2.33 & M \\
\hline
\end{tabular}

Note: $*=$ Observations recorded during the month of May-June; **= E, M and L indicate early, mid and late leaf flushing respectively; Data based on 10 ramets. 
In plot IX, where 37 MP teak clones were planted during the year 1982 (Table 13), it was observed that in the year 2002, 2 clones recorded as early leaf flushers, 13 as mid and 22 clones exhibited late leaf flushing. In 2003,2 clones showed early, 23 as mid leaf flushing and 12 clones as late leaf flushers. In 2004, 5 clones showed as early leaf flushing, $26 \mathrm{mid}$ and 6 clones as late leaf flushers. The average of three years observations revealed that 3 clones, viz. C-11 and PT-45, 47 as early, 22 clones, viz. C-6, 7, 8, 9, 10, 12, 14, 16, 17, 26, 30, 33, BHC-17, BLC-8, 11 and CSC-7, 8, 9, 11, 12, 26, 27 as mid, 12 clones, viz. C-15, 18, 42, 59, PT-3, BLC-1, 5, 6 and CSC-15, 17, 20, 25 as late leaf flushers.

Table 14. Observations on leaf flushing behaviour of 13 MP teak clones planted during the year 1985 in TSO, Behrai, Seoni (Plot X).

\begin{tabular}{lrrrrr}
\hline \multirow{2}{*}{$\begin{array}{l}\text { MP } \\
\text { teak clone }\end{array}$} & \multicolumn{4}{c}{ Leaf flushing } \\
\cline { 2 - 5 } & $\mathbf{2 0 0 2}$ & $\mathbf{2 0 0 3}$ & $\mathbf{2 0 0 4}$ & Mean & \multirow{2}{*}{ Period** $^{*}$} \\
\cline { 2 - 5 } C-1 & 3.00 & 2.00 & 2.00 & 2.33 & $\mathrm{M}$ \\
C-8 & 1.00 & 1.00 & 1.00 & 1.00 & $\mathrm{E}$ \\
C-17 & 2.00 & 2.00 & 2.00 & 2.00 & $\mathrm{M}$ \\
C-26 & 2.00 & 2.00 & 2.00 & 2.00 & $\mathrm{M}$ \\
C-34 & 3.00 & 2.00 & 2.00 & 2.33 & $\mathrm{M}$ \\
C-46 & 3.00 & 2.00 & 2.00 & 2.33 & $\mathrm{M}$ \\
C-47 & 3.00 & 3.00 & 2.00 & 2.67 & $\mathrm{~L}$ \\
C-49 & 3.00 & 3.00 & 3.00 & 3.00 & $\mathrm{~L}$ \\
C-53 & 2.00 & 2.00 & 2.00 & 2.00 & $\mathrm{M}$ \\
PT-1 & 1.00 & 1.00 & 1.00 & 1.00 & $\mathrm{E}$ \\
PT-3 & 2.00 & 2.00 & 2.00 & 2.00 & $\mathrm{M}$ \\
PT-45 & 2.00 & 1.00 & 1.00 & 1.33 & $\mathrm{E}$ \\
PT-46 & 2.00 & 2.00 & 1.00 & 1.67 & $\mathrm{M}$ \\
\hline Note & Observann &
\end{tabular}

Note: *= Observations recorded during the month of May-June; **=E, M and L indicate early, mid and late leaf flushing respectively; Data based on 10 ramets.

In plot X, where 13 MP teak clones were planted during the year 1985 (Table 14), it was recorded that in the year 2002, 2 clones recorded as early leaf flusher, 6 clones as mid and 5 as late leaf flushers. In 2003, 3 clones showed early leaf flushing, 8 clones as mid and 2 clones as late leaf flushers. In 2004, 4 clones showed early leaf flushing, 8 mid and only one clone as late leaf flushers. The average of three years observations revealed that 3 clones, viz. C-8 and PT-1, 45 as early, 8 clones, viz. C-1, 17, 26, 34, 46, 53 and PT-3, 46 mid and 2 clones, viz. C-47, 49 as late leaf flushers.

Table 15. Mean data on three years (2002-2004) observations of leaf flushing behaviour in 123 MP teak clones planted in TSO, Behrai, Seoni, in different years.

\begin{tabular}{|c|c|c|c|c|c|c|c|c|c|c|}
\hline \multirow{2}{*}{$\begin{array}{l}\text { MP } \\
\text { teak clone }\end{array}$} & \multicolumn{10}{|c|}{ Leaf flushing code in different plantation year } \\
\hline & 1977 & 1978 & $\begin{array}{r}1979 \\
\text { Part-I }\end{array}$ & $\begin{array}{r}1979 \\
\text { Part-II }\end{array}$ & $\begin{array}{r}1980 \\
\text { Part-I }\end{array}$ & $\begin{array}{r}1980 \\
\text { Part-II }\end{array}$ & $\begin{array}{r}1981 \\
\text { Part-I }\end{array}$ & $\begin{array}{r}1981 \\
\text { Part-II }\end{array}$ & 1982 & 1985 \\
\hline B-1 & 2.37 & & & & & & & & & \\
\hline C-1 & 2.20 & & 2.57 & & & & 2.63 & 2.4 & & 2.27 \\
\hline $\mathrm{C}-2$ & 1.73 & & 1.73 & & & & 1.67 & & & \\
\hline C-3 & 1.77 & 1.57 & 2.07 & & & & & & & \\
\hline C-4 & & 1.67 & & & 1.87 & & 1.73 & 1.87 & & \\
\hline C-5 & & 2.40 & 2.23 & & & & 2.33 & & & \\
\hline C-6 & 2.13 & & & & & & 2.43 & & 2.53 & \\
\hline C-7 & & & & & & & & & 2.47 & \\
\hline C-8 & 1.37 & & 1.90 & 1.83 & 1.47 & & & & 1.97 & 1.37 \\
\hline C-9 & 1.50 & & & & 1.67 & & & & 2.10 & \\
\hline C-10 & 2.43 & & & & 2.33 & & & & 2.37 & \\
\hline C-11 & 1.87 & 1.60 & & & 1.97 & & & & 1.67 & \\
\hline .C-12 & 2.27 & & & & & & & & 2.47 & \\
\hline C-14 & & & & & & & & & 2.43 & \\
\hline C-15 & & 2.33 & 2.17 & & & & & & 2.57 & \\
\hline C-16 & & & & & & & & & 2.47 & \\
\hline C-17 & & & 2.43 & & & & & & 2.27 & 2.67 \\
\hline C-18 & & & & & & & 2.43 & & 2.57 & \\
\hline C-19 & & & 2.17 & & & & 2.73 & & & \\
\hline C-20 & & & 2.23 & & & & & & & \\
\hline C-22 & & & 2.20 & 2.33 & & & & & & \\
\hline $\mathrm{C}-23$ & & & 2.23 & & & & & & & \\
\hline C-24 & & & 2.53 & & & & & & & \\
\hline
\end{tabular}


Plants and Environment (2020) 2(1): 13-30

\begin{tabular}{|c|c|c|c|c|c|c|c|c|c|c|}
\hline C-25 & & & 2.50 & 2.57 & 2.43 & & 2.83 & & & \\
\hline C-26 & & & 2.27 & 2.27 & 2.57 & & 2.57 & & 2.43 & 1.80 \\
\hline $\mathrm{C}-27$ & & & 2.17 & 2.37 & 2.43 & & & & & \\
\hline C- 28 & & & 2.17 & 2.47 & & 2.33 & & & & \\
\hline C-29 & & & & & & 2.63 & & & & \\
\hline C-30 & & & & & & & & & 2.47 & \\
\hline C-31 & & & & & & & & 2.43 & & \\
\hline C-32 & & & & & & 2.83 & & & & \\
\hline C-33 & & & & & & & & & 2.53 & \\
\hline C-34 & & & 2.53 & & & & 2.53 & 2.47 & & 2.17 \\
\hline C-35 & & & 2.30 & 2.40 & & 2.23 & & & & \\
\hline C-36 & & & & & & & 2.53 & & & \\
\hline C-37 & & & 2.13 & & & & & & & \\
\hline C-38 & & & 2.33 & 2.30 & 2.33 & & & & & \\
\hline C-39 & & & 2.07 & 2.27 & 2.57 & & & & & \\
\hline C-40 & & & 2.13 & 2.03 & 2.47 & & 1.67 & & & \\
\hline C-41 & & & 2.37 & & 2.23 & & 2.70 & & & \\
\hline C- 42 & & & 2.40 & & & & 2.77 & 2.57 & 2.77 & \\
\hline C-44 & & & 2.47 & 2.40 & & & & & & \\
\hline C-45 & & & 2.17 & 1.90 & 2.00 & 1.87 & 1.83 & 1.70 & & \\
\hline C-46 & & & 2.27 & 2.40 & & & & & & 2.43 \\
\hline C-47 & & & 2.33 & & & & 2.67 & 2.60 & & 2.57 \\
\hline C-48 & & & 2.23 & & & & 2.37 & & & \\
\hline C-49 & & & 2.10 & & 2.67 & & 2.73 & & & 2.97 \\
\hline C-50 & & & 2.30 & & 2.63 & & & & & \\
\hline C-51 & & & 2.10 & & & & & & & \\
\hline C-52 & & & & 2.53 & & & & & & \\
\hline C-53 & & & 2.17 & & & & & & & 2.87 \\
\hline C-54 & & & 2.20 & 2.10 & & & & & & \\
\hline C-56 & & & 2.50 & 2.67 & & & 2.73 & & & \\
\hline C-57 & & & 2.13 & & 2.23 & & 2.63 & & & \\
\hline C-59 & & & & & 2.70 & & & & 2.83 & \\
\hline$C-60$ & & & 1.77 & & 2.37 & & & & & \\
\hline F-1 & 2.03 & & 2.23 & & 1.83 & & & & & \\
\hline G-1 & 1.83 & & & & & & & & & \\
\hline K-1 & 1.77 & & & & 1.57 & & & & & \\
\hline M-1 & & & & & 2.37 & & & & & \\
\hline PT-1 & 1.83 & 1.63 & & & 1.93 & 1.97 & 1.93 & 2.03 & & 1.93 \\
\hline PT-3 & 2.27 & & & & & & & & 2.53 & 2.27 \\
\hline PT-5 & & & & & & & 2.43 & & & \\
\hline PT-6 & & & & & & & 2.37 & & & \\
\hline PT-7 & & & & & & & 2.93 & 2.33 & & \\
\hline PT-26 & 1.63 & & & & & & & & & \\
\hline PT-41 & 1.47 & & & & & & & & & \\
\hline PT-45 & 1.77 & 1.47 & & & & & & & 1.67 & 1.67 \\
\hline PT-46 & 1.63 & 1.57 & 1.77 & & & & & & & 1.63 \\
\hline PT-47 & & 1.57 & & & & & & & 1.97 & \\
\hline BBC-12 & & & & & 2.37 & & & & & \\
\hline BBC-14 & & & & & 2.67 & & & & & \\
\hline BBC-15 & & & & & 2.47 & 2.53 & & & & \\
\hline BHC-16 & & & & & 2.70 & & & & & \\
\hline BHC-17 & & & & & 2.63 & & & & 2.37 & \\
\hline BHC-18 & & & & & 2.67 & 2.83 & & & & \\
\hline BHC-19 & & & 2.03 & & 1.73 & 1.67 & & & & \\
\hline BLC-1 & & & & & & & & & 2.73 & \\
\hline BLC-5 & & & & & 2.93 & & & & 2.53 & \\
\hline BLC-6 & & & & & 2.70 & & & & 2.67 & \\
\hline BLC-7 & & & & & 2.93 & & & & & \\
\hline BLC-8 & & & 2.23 & & 2.53 & & & & 2.47 & \\
\hline BLC-10 & & & 2.47 & & & & & & & \\
\hline BLC-11 & & & & & & & & & 2.23 & \\
\hline CSC-1 & & & & & & & 2.57 & & & \\
\hline CSC-4 & & & & & & & 2.60 & & & \\
\hline
\end{tabular}




\begin{tabular}{|c|c|c|c|c|c|c|c|c|c|c|}
\hline CSC-5 & & & & & & & 2.37 & & & \\
\hline CSC-6 & & & & & & & 2.20 & & & \\
\hline CSC-7 & & & & & 2.77 & & & & 2.47 & \\
\hline CSC-8 & & & & & & & & & 2.60 & \\
\hline CSC-9 & & & & & 1.70 & 1.87 & 1.77 & & 1.87 & \\
\hline CSC-10 & & & & & 2.57 & & 2.60 & 2.30 & & \\
\hline CSC-11 & & & & & & & 2.53 & & 2.27 & \\
\hline CSC-12 & & & & & & & & & 2.67 & \\
\hline CSC-13 & & & & & & & 2.80 & & & \\
\hline CSC-14 & & & & & & 2.93 & 2.87 & & & \\
\hline CSC-15 & & & & & 2.43 & & & & 2.77 & \\
\hline CSC-16 & & & & & & & 2.77 & & & \\
\hline CSC-17 & & & & & 2.63 & & 2.67 & & 2.77 & \\
\hline CSC-18 & & & & & 2.63 & & 2.40 & & & \\
\hline CSC-19 & & & & & 2.73 & & & & & \\
\hline CSC-20 & & & & & 2.67 & & & & 2.60 & \\
\hline CSC-21 & & & & & 2.27 & & 2.57 & & & \\
\hline CSC-22 & & & & & & & 2.47 & & & \\
\hline CSC-23 & & & & & 2.53 & & 2.60 & & & \\
\hline CSC-24 & & & & & & & 2.60 & & & \\
\hline CSC-25 & & & & & & 2.27 & & & 2.63 & \\
\hline CSC-26 & & & & & & & & & 2.57 & \\
\hline CSC-27 & & & & & & & & & 2.57 & \\
\hline CSC-28 & & & & & 2.63 & & & & & \\
\hline CSC-29 & & & & & & & 2.27 & & & \\
\hline CSC-30 & & & & & & & 2.27 & & & \\
\hline CSC-31 & & & & & & & 2.37 & & & \\
\hline CSC-32 & & & & & 2.43 & & & & & \\
\hline CSC-60 & & & & & 2.57 & & & & & \\
\hline MBC-4 & & & & & & & 2.53 & 2.47 & & \\
\hline MKC-24 & & & & & & & & 2.57 & & \\
\hline MKC-25 & & & & & & & 2.53 & & & \\
\hline MTC-6 & & & & & & & 2.47 & 2.43 & & \\
\hline MTC-7 & & & & & & & & 2.33 & & \\
\hline MTC-8 & & & & & & & 2.57 & & & \\
\hline MTC-12 & & & & & & & & 2.27 & & \\
\hline MTC-27 & & & & & & & 2.53 & & & \\
\hline \multicolumn{11}{|c|}{ Within the year } \\
\hline Average & 1.89 & 1.76 & 2.22 & 2.30 & 2.38 & 2.33 & 2.46 & 2.32 & 2.43 & 2.20 \\
\hline SEm & 0.183 & 0.162 & 0.186 & 0.172 & 0.184 & 0.164 & 0.169 & 0.193 & 0.179 & 0.186 \\
\hline $\begin{array}{r}\text { C.D. at } \\
5 \%\end{array}$ & 0.507 & 0.455 & 0.514 & 0.477 & 0.509 & 0.463 & 0.467 & 0.536 & 0.496 & 0.523 \\
\hline $\begin{array}{r}\text { C.D. at } \\
1 \%\end{array}$ & 0.667 & 0.603 & 0.676 & 0.605 & 0.668 & 0.613 & 0.614 & 0.692 & 0.652 & 0.693 \\
\hline \multicolumn{11}{|c|}{ Over the year } \\
\hline SEm & 0.156 & 0.066 & 0.161 & 0.134 & 0.105 & 0.089 & 0.101 & 0.137 & 0.095 & 0.099 \\
\hline $\begin{array}{r}\text { C.D. at } \\
5 \%\end{array}$ & 0.449 & 0.197 & 0.454 & 0.386 & 0.295 & 0.261 & 0.285 & 0.397 & 0.268 & 0.290 \\
\hline $\begin{array}{r}\text { C.D. at } \\
1 \%\end{array}$ & 0.601 & 0.271 & NS & 0.520 & 0.392 & 0.355 & 0.378 & 0.536 & 0.355 & 0.393 \\
\hline
\end{tabular}

Note: *Number of clones 123; SEm= Standard error mean; $\mathrm{CD}=$ Critical difference.

The observations recorded during the three years of study (2002-2004) in 10 experimental plots in TSO, Behrai, Seoni (Table 15) revealed that leaf flushing in MP teak clones occurs in the month of May-June, irrespective of clones and years (Table 16). It was noticed that the variation in leaf flushing periods within the clones and among the clones of different localities were found to be significantly $(\mathrm{P}<0.05-\mathrm{P}<0.01)$ heterogeneous within and over the years.

Table 16. Pooled data on three years (2002-2004) observations of leaf flushing behaviour in MP teak clones planted in TSO, Behrai, Seoni.

\begin{tabular}{lrrrrr}
\hline MP & \multicolumn{2}{l}{ Leaf flushing code in different year } & Pooled mean & $\begin{array}{r}\text { Leaf flushing } \\
\text { period }\end{array}$ \\
\cline { 2 - 4 } & $\mathbf{2 0 0 2}$ & $\mathbf{2 0 0 3}$ & $\mathbf{2 0 0 4}$ & & $\mathrm{M}$ \\
\hline B-1 & 2.30 & 2.50 & 2.30 & 2.37 & $\mathrm{~L}$ \\
$\mathrm{C}-1$ & 2.56 & 2.34 & 2.34 & 2.41 & $\mathrm{E}$ \\
$\mathrm{C}-2$ & 2.10 & 1.57 & 1.47 & 1.71 & \\
\hline
\end{tabular}


Plants and Environment (2020) 2(1): 13-30

\begin{tabular}{|c|c|c|c|c|c|}
\hline C-3 & 1.87 & 2.00 & 1.53 & 1.80 & $\mathrm{E}$ \\
\hline C-4 & 2.00 & 1.63 & 1.73 & 1.78 & E \\
\hline C-5 & 2.40 & 2.23 & 2.33 & 2.32 & $\mathrm{M}$ \\
\hline C-6 & 2.57 & 2.27 & 2.27 & 2.37 & M \\
\hline C-7 & 2.30 & 2.40 & 2.70 & 2.47 & M \\
\hline C-8 & 1.75 & 1.60 & 1.60 & 1.65 & E \\
\hline C-9 & 1.93 & 1.63 & 1.70 & 1.76 & $\mathrm{E}$ \\
\hline C-10 & 2.47 & 2.47 & 2.20 & 2.38 & M \\
\hline C-11 & 1.85 & 1.88 & 1.60 & 1.78 & E \\
\hline C-12 & 2.35 & 2.40 & 2.35 & 2.37 & M \\
\hline C-14 & 2.40 & 2.60 & 2.30 & 2.43 & $\mathrm{M}$ \\
\hline C-15 & 2.33 & 2.40 & 2.33 & 2.36 & M \\
\hline C-16 & 2.70 & 2.40 & 2.30 & 2.47 & $\mathrm{M}$ \\
\hline C-17 & 2.53 & 2.50 & 2.33 & 2.46 & $\mathrm{M}$ \\
\hline C-18 & 2.60 & 2.40 & 2.50 & 2.50 & M \\
\hline C-19 & 2.35 & 2.50 & 2.50 & 2.45 & $\mathrm{~L}$ \\
\hline C-20 & 2.40 & 2.10 & 2.20 & 2.23 & M \\
\hline C-22 & 2.30 & 2.15 & 2.35 & 2.27 & M \\
\hline C-23 & 2.30 & 2.20 & 2.20 & 2.23 & $\mathrm{M}$ \\
\hline C-24 & 2.50 & 2.50 & 2.60 & 2.53 & $\mathrm{M}$ \\
\hline C-25 & 2.73 & 2.53 & 2.50 & 2.58 & $\mathrm{~L}$ \\
\hline C-26 & 2.35 & 2.28 & 2.32 & 2.32 & M \\
\hline C-27 & 2.33 & 2.27 & 2.37 & 2.32 & $\mathrm{M}$ \\
\hline C-28 & 2.50 & 2.30 & 2.17 & 2.32 & $\mathrm{M}$ \\
\hline C-29 & 2.80 & 2.50 & 2.60 & 2.63 & M \\
\hline C-30 & 2.70 & 2.20 & 2.50 & 2.47 & $\mathrm{M}$ \\
\hline C-31 & 2.50 & 2.10 & 2.70 & 2.43 & M \\
\hline C-32 & 2.80 & 2.90 & 2.80 & 2.83 & $\mathrm{M}$ \\
\hline C-33 & 2.60 & 2.60 & 2.40 & 2.53 & $\mathrm{M}$ \\
\hline C-34 & 2.60 & 2.35 & 2.33 & 2.43 & $\mathrm{~L}$ \\
\hline C-35 & 2.43 & 2.30 & 2.20 & 2.31 & $\mathrm{M}$ \\
\hline C-36 & 2.60 & 2.60 & 2.40 & 2.53 & M \\
\hline C-37 & 2.20 & 2.20 & 2.00 & 2.13 & M \\
\hline C-38 & 2.50 & 2.27 & 2.20 & 2.32 & $\mathrm{M}$ \\
\hline C-39 & 2.27 & 2.30 & 2.33 & 2.30 & M \\
\hline C-40 & 1.98 & 2.20 & 2.05 & 2.08 & M \\
\hline C-41 & 2.67 & 2.47 & 2.17 & 2.43 & $\mathrm{~L}$ \\
\hline C-42 & 2.78 & 2.50 & 2.60 & 2.63 & $\mathrm{~L}$ \\
\hline C-44 & 2.65 & 2.10 & 2.55 & 2.43 & $\mathrm{~L}$ \\
\hline C-45 & 1.80 & 1.98 & 1.95 & 1.91 & M \\
\hline C-46 & 2.57 & 2.37 & 2.17 & 2.37 & M \\
\hline C-47 & 2.65 & 2.50 & 2.48 & 2.54 & $\mathrm{M}$ \\
\hline C- 48 & 2.10 & 2.50 & 2.30 & 2.30 & M \\
\hline C-49 & 2.58 & 2.75 & 2.53 & 2.62 & $\mathrm{~L}$ \\
\hline C-50 & 2.35 & 2.55 & 2.50 & 2.47 & $\mathrm{M}$ \\
\hline C-51 & 2.00 & 2.20 & 2.10 & 2.10 & $\mathrm{M}$ \\
\hline C-52 & 2.40 & 2.60 & 2.60 & 2.53 & M \\
\hline C-53 & 2.70 & 2.40 & 2.45 & 2.52 & M \\
\hline C-54 & 1.90 & 2.20 & 2.35 & 2.15 & E \\
\hline C-56 & 2.73 & 2.57 & 2.60 & 2.63 & $\mathrm{~L}$ \\
\hline C-57 & 2.33 & 2.37 & 2.30 & 2.33 & M \\
\hline C-59 & 2.85 & 2.75 & 2.70 & 2.77 & $\mathrm{~L}$ \\
\hline C-60 & 2.30 & 2.15 & 1.75 & 2.07 & $\mathrm{M}$ \\
\hline F-1 & 2.23 & 1.87 & 2.00 & 2.03 & E \\
\hline G-1 & 1.70 & 1.80 & 2.00 & 1.83 & E \\
\hline $\mathrm{K}-1$ & 2.05 & 1.40 & 1.55 & 1.67 & E \\
\hline M-1 & 2.60 & 2.30 & 2.20 & 2.37 & M \\
\hline PT-1 & 2.10 & 1.80 & 1.79 & 1.90 & E \\
\hline PT-3 & 2.53 & 2.27 & 2.27 & 2.36 & M \\
\hline PT-5 & 2.30 & 2.80 & 2.20 & 2.43 & M \\
\hline PT-6 & 2.70 & 2.30 & 2.10 & 2.37 & M \\
\hline PT-7 & 2.70 & 2.65 & 2.55 & 2.63 & $\mathrm{~L}$ \\
\hline PT-26 & 2.00 & 1.30 & 1.60 & 1.63 & $\mathrm{E}$ \\
\hline
\end{tabular}




\begin{tabular}{|c|c|c|c|c|c|}
\hline PT-41 & 1.80 & 1.50 & 1.10 & 1.47 & $\mathrm{E}$ \\
\hline PT-45 & 1.78 & 1.78 & 1.38 & 1.64 & $\mathrm{E}$ \\
\hline PT-46 & 1.85 & 1.68 & 1.43 & 1.65 & $\mathrm{E}$ \\
\hline PT-47 & 1.80 & 1.65 & 1.85 & 1.77 & $\mathrm{E}$ \\
\hline BBC-12 & 2.50 & 2.30 & 2.30 & 2.37 & M \\
\hline BBC-14 & 2.70 & 2.80 & 2.50 & 2.67 & $\mathrm{~L}$ \\
\hline BBC-15 & 2.55 & 2.50 & 2.45 & 2.50 & M \\
\hline BHC-16 & 2.90 & 2.50 & 2.70 & 2.70 & M \\
\hline BHC-17 & 2.40 & 2.55 & 2.55 & 2.50 & M \\
\hline BHC-18 & 2.80 & 2.80 & 2.65 & 2.75 & $\mathrm{~L}$ \\
\hline BHC-19 & 2.20 & 1.67 & 1.57 & 1.81 & $\mathrm{E}$ \\
\hline BLC-1 & 2.80 & 2.80 & 2.60 & 2.73 & $\mathrm{~L}$ \\
\hline BLC-5 & 2.90 & 2.55 & 2.75 & 2.73 & M \\
\hline BLC-6 & 2.65 & 2.75 & 2.65 & 2.68 & $\mathrm{~L}$ \\
\hline BLC-7 & 2.90 & 2.90 & 3.00 & 2.93 & $\mathrm{~L}$ \\
\hline BLC-8 & 2.33 & 2.43 & 2.47 & 2.41 & $\mathrm{~L}$ \\
\hline BLC-10 & 3.00 & 2.50 & 1.90 & 2.47 & $\mathrm{~L}$ \\
\hline BLC-11 & 2.20 & 2.20 & 2.30 & 2.23 & $\mathrm{M}$ \\
\hline CSC-1 & 2.60 & 2.80 & 2.30 & 2.57 & $\mathrm{M}$ \\
\hline CSC-4 & 2.70 & 2.50 & 2.60 & 2.60 & M \\
\hline CSC-5 & 2.50 & 2.10 & 2.50 & 2.37 & $\mathrm{M}$ \\
\hline CSC-6 & 2.30 & 1.90 & 2.40 & 2.20 & $\mathrm{M}$ \\
\hline CSC-7 & 2.65 & 2.60 & 2.60 & 2.62 & $\mathrm{~L}$ \\
\hline CSC-8 & 2.80 & 2.60 & 2.40 & 2.60 & $\mathrm{M}$ \\
\hline CSC-9 & 1.88 & 2.08 & 1.45 & 1.80 & E \\
\hline CSC-10 & 2.47 & 2.47 & 2.53 & 2.49 & $\mathrm{~L}$ \\
\hline CSC-11 & 2.40 & 2.45 & 2.35 & 2.40 & $\mathrm{~L}$ \\
\hline CSC-12 & 2.80 & 2.60 & 2.60 & 2.67 & M \\
\hline CSC-13 & 2.80 & 2.90 & 2.70 & 2.80 & $\mathrm{~L}$ \\
\hline CSC-14 & 2.95 & 2.95 & 2.80 & 2.90 & $\mathrm{~L}$ \\
\hline CSC-15 & 2.50 & 2.70 & 2.60 & 2.60 & $\mathrm{~L}$ \\
\hline CSC-16 & 2.90 & 2.80 & 2.60 & 2.77 & $\mathrm{~L}$ \\
\hline CSC-17 & 2.73 & 2.70 & 2.63 & 2.69 & $\mathrm{~L}$ \\
\hline CSC-18 & 2.65 & 2.45 & 2.45 & 2.52 & $\mathrm{M}$ \\
\hline CSC-19 & 2.70 & 2.60 & 2.90 & 2.73 & $\mathrm{M}$ \\
\hline CSC-20 & 2.80 & 2.55 & 2.55 & 2.63 & $\mathrm{M}$ \\
\hline CSC-21 & 2.60 & 2.40 & 2.25 & 2.42 & $\mathrm{M}$ \\
\hline CSC-22 & 2.40 & 2.60 & 2.40 & 2.47 & $\mathrm{M}$ \\
\hline CSC-23 & 2.55 & 2.55 & 2.60 & 2.57 & $\mathrm{M}$ \\
\hline CSC-24 & 2.60 & 2.70 & 2.50 & 2.60 & $\mathrm{M}$ \\
\hline CSC-25 & 2.50 & 2.40 & 2.45 & 2.45 & $\mathrm{M}$ \\
\hline CSC-26 & 2.70 & 2.40 & 2.60 & 2.57 & $\mathrm{M}$ \\
\hline CSC-27 & 2.70 & 2.60 & 2.40 & 2.57 & $\mathrm{M}$ \\
\hline CSC-28 & 2.90 & 2.60 & 2.40 & 2.63 & $\mathrm{~L}$ \\
\hline CSC-29 & 2.10 & 2.10 & 2.60 & 2.27 & $\mathrm{M}$ \\
\hline CSC-30 & 2.60 & 2.10 & 2.10 & 2.27 & M \\
\hline CSC-31 & 2.60 & 2.40 & 2.10 & 2.37 & M \\
\hline CSC-32 & 2.70 & 2.50 & 2.10 & 2.43 & $\mathrm{M}$ \\
\hline CSC-60 & 2.60 & 2.70 & 2.40 & 2.57 & M \\
\hline MBC-4 & 2.50 & 2.35 & 2.65 & 2.50 & M \\
\hline MKC-24 & 2.40 & 2.60 & 2.70 & 2.57 & $\mathrm{M}$ \\
\hline MKC-25 & 2.70 & 2.30 & 2.60 & 2.53 & M \\
\hline MTC-6 & 2.35 & 2.40 & 2.60 & 2.45 & M \\
\hline MTC-7 & 2.10 & 2.20 & 2.70 & 2.33 & $\mathrm{M}$ \\
\hline MTC-8 & 2.50 & 2.50 & 2.70 & 2.57 & M \\
\hline MTC-12 & 2.10 & 2.30 & 2.40 & 2.27 & M \\
\hline MTC-27 & 2.70 & 2.40 & 2.50 & 2.53 & $\mathrm{M}$ \\
\hline Average & 2.45 & 2.35 & 2.32 & 2.37 & - \\
\hline SEm & 0.176 & 0.182 & 0.175 & 0.097 & - \\
\hline C.D. at $5 \%$ & 0.490 & 0.507 & 0.487 & 0.269 & - \\
\hline C.D. at $1 \%$ & 0.646 & 0.660 & 0.641 & 0.353 & - \\
\hline
\end{tabular}

Note: *Number of clones 123 ; $\mathrm{SEm}=$ Standard error mean; $\mathrm{CD}=$ Critical difference; $* * \mathrm{E}, \mathrm{M}$ and $\mathrm{L}$ indicate early, mid and late leaf flushing respectively. 
Out of 123 MP teak clones that were planted during the year 1977-1985, 18 clones showed early leaf flushing (16$31^{\text {st }}$ May) followed by 78 clones as mid ( $1^{\text {st }}$ June to $15^{\text {th }}$ June) and 27 as late leaf flushers ( $16^{\text {th }}$ June to $30^{\text {th }}$ June) (Table 17) (Fig. 3). These early leaf flushing teak clones noticed were C-2, 3, 4, 8, 9, 11, 54, F-1, G-1, K-1, PT-1, 26, 41, 45, 46, 47, BHC-19 and CSC-9. The mid leaf flushing teak clones recorded were B-1, C-5, 6, 7, 10, 12, 14, 15, 16, 17, 18, 20, 22, 23, 24, 26, 27, 28, 29, 30, 31, 32, 33, 35, 36, 37, 38, 39, 40, 45, 46, 47, 48, 50, 51, 52, 53, 57, 60, M-1, PT-3, 5, 6, BBC-12, 15, BHC-16, 17, BLC-5, 11, CSC-1, 4, 5, 6, 8, 12, 18, 19, 20, 21, 22, 23, 24, 25, 26, 27, 29, 30, 31, 32, 60, MBC-4, MKC-24, 25 and MTC-6, 7, 8, 12, 27. The late leaf flushers teak clones observed were C-1, 19, 25, 34, 41, 42, 44, 49, 56, 59, PT-7, BBC-14, BHC-18, BLC-1, 6, 7, 8, 10 and CSC-7, 10, 11, 13, 14, 15, 16, 17, 28.

Table 17. Leaf flushing period of MP teak clones planted in TSO, Behrai, Seoni, Madhya Pradesh.

\begin{tabular}{llr}
\hline Category & MP teak clones & Total \\
\hline $\begin{array}{l}\text { Early } \\
\text { (May 16 - May 31) }\end{array}$ & C-2, 3, 4, 8, 9, 11, 54, F-1, G-1, K-1, PT-1, 26, 41, 45, 46, 47, BHC-19, CSC-9 & 18 \\
Mid & B-1, C-5, 6, 7, 10, 12, 14, 15, 16, 17, 18, 20, 22, 23, 24, 26, 27, 28, 29, 30, 31, 32, & 78 \\
(June 01 - June 15) & $33,35,36,37,38,39,40,45,46,47,48,50,51,52,53,57,60$, M-1, PT-3, 5, 6, & \\
& BBC-12, 15, BHC-16, 17, BLC-5, 11, CSC-1, 4, 5, 6, 8, 12, 18, 19, 20, 21, 22, 23, & \\
& $24,25,26,27,29,30,31,32,60$, MBC-4, MKC-24, 25, MTC-6, 7, 8, 12, 27 & 27 \\
Late & C-1, 19, 25, 34, 41, 42, 44, 49, 56, 59, PT-7, BBC-14, BHC-18, BLC-1, 6, 7, 8, & 27 \\
(June 16 - June 30) & 10, CSC-7, 10, 11, 13, 14, 15, 16, 17, 28 & $\mathbf{1 2 3}$ \\
\hline Total & & \\
\hline
\end{tabular}

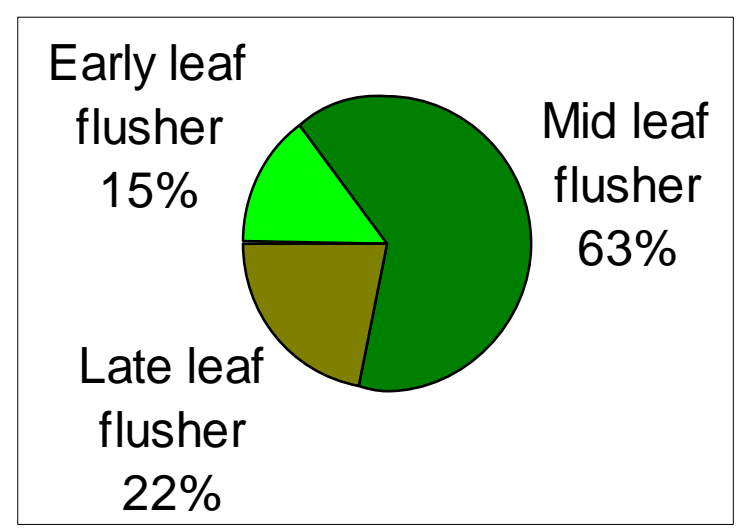

Figure 3. Percentage occurrence of MP teak clones in respect of leaf flushing period in TSO, Behrai, Seoni, Madhya Pradesh.

\section{DISCUSSION}

Leaf flushing is the production of a flush of new leaves typically produced simultaneously on all branches of a bare plant or tree. The observations recorded during the three years of study on leaf flushing behaviour as a parameter of morphological study in 123 teak clones of MP origin, planted in different year in TSO, Behrai, Seoni, have exhibited that leaf flushing in MP teak occur in the month of May-June. According to a phonological study performed by Priya \& Bhat (1998) in Kerala made on the teak plantation of three different localities, such as Nilambur, Walayar and Peechi for two consecutive years during 1994-95, leaf flushing in teak occurs in the month of March-April. The authors have also suggested that cambial activity is responsible for leaf flushing in teak. However, the observation as recorded in the present study is in partial agreement with the findings of Ansari et al. (2001), who have also conducted a systematic phenological study in teak forests of Madhya Pradesh and suggested that teak grows actively, producing new flush of leaves during the period from April-June. The observations of the present study have also shown variations in period of leaf flushing in respect of teak clones. Out of 123 MP teak clones planted in TSO, Behrai, Seoni, only 18 clones showed early leaf flushing whereas 78 clones as mid and 27 clones as late leaf flushers. The differential behaviour of clones in respect of leaf flushing period clearly suggests that it is mainly due to the variations in genotypes of teak. The present findings corroborate the observations of Nair et al. (1989), who have also recorded wide variation in the leaf flushing period among teak clones of Kerala origin, including 'teli' variety of teak and its relevant species, Tectona hamiltoniana Wall.

\section{ACKNOWLEDGEMENTS}

Authors are thankful to Director, Tropical Forest Research Institute, Jabalpur-482021 (M.P.), for providing necessary research facilities to carry out this work under the extramural research project, entitled "Screening and identification of teak of Madhya Pradesh for resistance against major insect pests" [ID No. 034/TFRI-2001/Ento- 
1(MPCST)(4)]. Authors are also grateful to Madhya Pradesh Council of Science and Technology (MPCST), Bhopal, for funding the research project.

\section{REFERENCES}

Ansari S.A., Kumar S., Sharma S. \& Shirin F. (2001). Clonal Propagation of Teak. In: Mandal A.K. \& Ansari S.A. (Eds.) Genetics and Silviculture of Teak. International Book Distributors, Dehradun, India, pp. 165-186.

Bhat K.M., Nair K.K.N., Bhat K.V., Muralidharan E.M. \& Sharma J.K. (2005). Quality Timber Products of Teak from Sustainable Forest Management. Kerala Forest Research Institute, Peechi, Kerala, India and International Tropical Timber Organization, Yokohama, Japan, 669 p.

Gangopadhyay P.B. (2005). A Report on Teak in Madhya Pradesh with Technical Analysis. In: Bhat K.M., Nair K.K.N., Bhat K.V., Muralidharan E.M. \& Sharma J.K. (Eds.) Quality Timber Products of Teak from Sustainable Forest Management. Kerala Forest Research Institute, Peechi, Kerala, India and International Tropical Timber Organization, Yokohama, Japan, pp. 24-30.

Gomez K.A. \& Gomez A.A. (1984). Statistical Procedures for Agricultural Research. John Wiley and Sons, New York, USA, 680 p.

Hedegart T. (1975). Breeding Systems, Variation and Genetic Improvement of Teak (Tectona grandis Linn.f.). In : Burley J. \& Styles B.T.(Eds.) Tropical Trees Variation, Breeding and Conservation. Academic Press, New York, USA, pp. 109-121.

Kumaravelu G. (1992). Teak in India. In: Wood H. (Ed.) Teak in Asia. FAO, Bangkok, pp. 27-34

Nair K.S.S., Kedharnath S., Koshy M.P., Mathew P. \& Sudheendrakumar V.V. (1989). Search for natural resistance to the insect pest, Hyblaea puera in teak. Kerala Forest Research Institute, Kerala, India, Research Report No. 62: 32 p.

Pande D. (1983). Growth and Yield of Plantation Species in the Tropics. F.A.O., Rome, $406 \mathrm{p}$

Priya P.B. \& Bhat K.M. (1998). Influence of rainfall, irrigation and age on the growth periodicity and wood structure in teak (Tectona grandis). IAWA Journal, 20: 181-192.

Roychoudhury N. (2016). Teak seed orchards and seed production in Madhya Pradesh. Vaniki Sandesh, 7: 11-24.

Roychoudhury N., Joshi K.C. \& Shukla N.P. (2003). Teak in Madhya Pradesh. ENVIS Forestry Bulletin, 3: 29-33.

Seth S.K. \& Kaul O.N. (1978). Tropical Forest Ecosystems of India: The teak forests. In: Tropical Forest Ecosystems. UNESCO, Paris, pp. 628-640.

Shukla P.K., Srivastava R., Jalil P. \& Sharama A. (2003). Tree improvement of teak in Madhaya Pradesh (India). Vaniki Sandesh, 27: 1-9.

Tiwari K.P., Sharma M.C. \& Jalil P. (1998a). Seed Production in Teak Seed Orchards in Madhya Pradesh (India). State Forest Research Institute, Jabalpur, India, Technical Bulletin No. 35: 55 p.

Tiwari K.P., Sharma M.C. \& Panday R.L. (1998b). Yield and Stand Tables of Teak in Madhya Pradesh. State Forest Research Institute, Jabalpur, India, Technical Bulletin No. 39: 85 p. 